KEK-TH-336

KEK preprint 92-42

hep-th/9206110

June 1992

\title{
Higher-Rank Supersymmetric Models and Topological Conformal Field Theory
}

\author{
Toshiya Kawai*, Taku Uchino ${ }^{\dagger}$ and Sung-Kil Yang $\$$ \\ * National Laboratory for High Energy Physics (KEK) \\ Tsukuba, Ibaraki 305, Japan \\ ${ }^{\dagger}$ Department of Physics, Tokyo Institute of Technology, \\ Oh-okayama, Meguro-ku, Tokyo 152, Japan \\ ${ }^{\ddagger}$ Institute of Physics, University of Tsukuba, \\ Tsukuba, Ibaraki 305, Japan
}

\begin{abstract}
In the first part of this paper we investigate the operator aspect of higher-rank supersymmetric model which is introduced as a Lie theoretic extension of the $N=2$ minimal model with the simplest case $s u(2)$ corresponding to the $N=2$ minimal model. In particular we identify the analogs of chirality conditions and chiral ring. In the second part we construct a class of topological conformal field theories starting with this higher-rank supersymmetric model. We show the BRST-exactness of the twisted stress-energy tensor, find out physical observables and discuss how to make their correlation functions. It is emphasized that in the case of $s u(2)$ the topological field theory constructed in this paper is distinct from the one obtained by twisting the $N=2$ minimal model through the usual procedure.
\end{abstract}

\$ Partially supported by Grant-in-Aid for Scientific Research on Priority Area 231 "Infinite Analysis". 


\section{Introduction}

Over the past several years the $N=2$ minimal model [1] has been a useful testing grounds for various theoretical ideas, especially those of geometrical and topological origins. It is natural to ask whether one can invent any interesting generalizations of the $N=2$ minimal model and, if such generalizations ever exist, what kind of insights one can gain from them. As discussed in [2,3], it is not difficult to imagine a class of models described by a set of free bosons and parafermions associated with an arbitrary simple Lie algebra $\mathbf{g}$ which reduce to the $N=2$ minimal model when specialized to $\mathbf{g}=s u(2)$. The approach taken there was primarily through branching relations. A simple analysis on these relations suggested the existence of 'supersymmetry' generalizing $N=2$ superconformal symmetry and the spectra of (analogs of) chiral primary states and the Ramond ground states were derived. We will refer to these models as higher-rank supersymmetric models.

The first aim of this article is to elaborate on the operator aspect of these models. We first review some basic properties of the WZW model and the associated parafermions in sect.2. In sect.3, after recalling the construction of the models, we consider the structure of chiral algebra and spectral flow. Then we investigate the chiral primary state conditions and the chiral ring together with their connections to the Ramond ground states. These results are mostly direct generalizations of the essential features of the $N=2$ minimal model, however, we will find that some properties observed in the $N=2$ minimal model are due to the specific nature of $s u(2)$.

One of the important characteristics of the $N=2$ minimal model is that they can be 'twisted' yielding a class of topological field theories [4] as discussed in [5]. This line of thought was further pursued in [6, [7]. Furthermore, it was investigated in [3] for $N=2$ hermitian symmetric space models how the twisting procedure can be seen at the level of characters. A natural question would be whether we can similarly make topological field theory out of our higher-rank supersymmetric model. Investigating this problem is the second aim of the paper. As it turns out in sect.4 the answer to this question is a

qualified yes: topological field theory we are able to construct for an arbitrary $\mathbf{g}$ is not strictly of the type we usually associate with the $N=2$ minimal model. Our reasoning rests critically on the BRST-exact expression of the twisted stress-energy tensor originally proposed in [7] in the context of $c=0$ GKO coset models. Actually a large portion of this paper is concerned with the (partial) proof of this formula. The next step is to seek BRST invariant physical observables and study their correlation functions. To produce 
viable correlation functions among the basic physical observables we have to introduce extra BRST invariant operators. As a consequence of this the topological conformal field theory we consider here for $\mathbf{g}=s u(2)$ is distinct from the one for the $N=2$ minimal model in the ordinary sense. We then comment on perturbations of our topological field theory and finally will speculate on the relation to $G / G$ theory [8].

In sect.5 concluding remarks are given while appendix contains several formulas to be used in sect.4 in evaluating multiple integrals.

A preliminary account of this work was reported in [9].

\section{WZW model and Parafermions.}

In this section we briefly summarize some basic properties of the WZW model and the associated parafermions [10 12] for the sake of later convenience.

We start with fixing our notation for Lie algebras. Let $\mathbf{g}$ be a finite-dimensional simple Lie algebra of rank $n$ and let $\alpha_{1}, \ldots, \alpha_{n}$ be its simple roots. The set of positive roots of $\mathrm{g}$ is denoted by $\Delta_{+}$so that $\Delta=\Delta_{+} \cup\left(-\Delta_{+}\right)$is the whole set of roots. We always follow the convention such that any long root of $\mathbf{g}$ has length $\sqrt{2}$ and hence $\theta^{2}=2$ where $\theta$ is the highest root of $\mathbf{g}$. For any root $\alpha$ its coroot is defined by $\alpha^{\vee}=\frac{2}{\alpha^{2}} \alpha$. Define a symmetric matrix $G$ by $(G)_{i j}=\alpha_{i}^{\vee} \cdot \alpha_{j}^{\vee}$. The fundamental weights $\omega_{1}, \ldots, \omega_{n}$ are dual to the simple coroots: $\omega_{i} \cdot \alpha_{j}^{\vee}=\delta_{i j}$. Let $P$ be the weight lattice of $\mathbf{g}$ and let $M$ be the coroot lattice of g. The Weyl vector $\rho$ is defined to be half the sum of the positive roots.

The WZW model at level $k$, where $k$ is a positive integer, is algebraically described by the integrable representations at level $k$ of the (untwisted) affine Kac-Moody algebra associated with $\mathbf{g}$. In the Chevalley basis the Kac-Moody algebra is determined by the mutual operator product expansions of $H_{i}(z), i=1, \ldots, n$ and $E_{\alpha}(z), \alpha \in \Delta$ :

$$
\begin{aligned}
& H_{i}(z) H_{j}\left(z^{\prime}\right)=\frac{k(G)_{i j}}{\left(z-z^{\prime}\right)^{2}}+\cdots, \\
& H_{i}(z) E_{\alpha}\left(z^{\prime}\right)=\frac{\left(\alpha \cdot \alpha_{i}^{\vee}\right)}{z-z^{\prime}} E_{\alpha}\left(z^{\prime}\right)+\cdots, \\
& E_{\alpha}(z) E_{-\alpha}\left(z^{\prime}\right)=\frac{k\left(2 / \alpha^{2}\right)}{\left(z-z^{\prime}\right)^{2}}+\frac{1}{z-z^{\prime}} \sum_{i=1}^{n} \nu_{i} H_{i}\left(z^{\prime}\right)+\cdots, \\
& E_{\alpha}(z) E_{\beta}\left(z^{\prime}\right)=\frac{N_{\alpha, \beta}}{z-z^{\prime}} E_{\alpha+\beta}\left(z^{\prime}\right)+\cdots
\end{aligned}
$$


In eq.(2.1d) $\nu_{i}$ 's are the integers such that $\alpha^{\vee}=\sum_{i=1}^{n} \nu_{i} \alpha_{i}^{\vee}$ and in eq.(2.1d) $N_{\alpha, \beta}=0$ if $\alpha+\beta \notin \Delta$ and $N_{\alpha, \beta}= \pm(s+1)$ if $\alpha+\beta \in \Delta$ where $s$ is the largest integer such that $\alpha-s \beta \in \Delta$.

The stress-energy tensor of the WZW model in the Sugawara form is

$$
T_{\text {Sug }}(z)=\frac{1}{2(k+g)}\left[\sum_{i, j=1}^{n}\left(G^{-1}\right)_{i j} \cdot H_{i}(z) H_{j}(z)^{*}+\sum_{\alpha \in \Delta} \frac{\alpha^{2}}{2}: E_{\alpha}(z) E_{-\alpha}(z)^{*} \cdot\right] \text {, }
$$

where $g$ is the dual Coxeter number of $\mathbf{g}$. As is well-known, the moments of $T_{\text {Sug }}(z)$ generate the Virasoro algebra with a central charge

$$
c=\frac{k d}{k+g}
$$

where $d=\operatorname{dim} \mathbf{g}$.

The generators of the Kac-Moody algebra can be realized in terms of free bosons $\varphi(z)=\left(\varphi_{1}(z), \ldots, \varphi_{n}(z)\right)$ and parafermions $\psi_{\alpha}(z), \alpha \in \Delta$ [1],12]:

$$
\begin{aligned}
& H_{i}(z)=i \sqrt{k} \alpha_{i}^{\vee} \cdot \partial \varphi(z), \quad i=1, \ldots, n, \\
& E_{\alpha}(z)=\sqrt{\frac{2 k}{\alpha^{2}}} \psi_{\alpha}(z)^{-} \cdot \exp [i \alpha \cdot \varphi(z) / \sqrt{k}] \cdot c_{\alpha}, \quad \alpha \in \Delta,
\end{aligned}
$$

where the cocycle factors $c_{\alpha}$ commute with $\varphi(z)$ and $\psi_{\alpha}(z)$, satisfying

$$
c_{\alpha} c_{\beta}=\epsilon(\alpha, \beta) c_{\alpha+\beta}, \quad \epsilon(\alpha, \beta)= \pm 1
$$

with

$$
\begin{aligned}
& \epsilon(\alpha, \beta) \epsilon(\alpha+\beta, \gamma)=\epsilon(\alpha, \beta+\gamma) \epsilon(\beta, \gamma), \\
& \epsilon(\alpha, \beta)=(-1)^{\alpha \cdot \beta+\alpha^{2} \beta^{2}} \epsilon(\beta, \alpha) .
\end{aligned}
$$

The OPEs of the free bosons and the parafermions are

$$
\begin{aligned}
& \varphi_{a}(z) \varphi_{b}\left(z^{\prime}\right)=-\delta_{a b} \log \left(z-z^{\prime}\right)+\cdots, \\
& \psi_{\alpha}(z) \psi_{-\alpha}\left(z^{\prime}\right)=\frac{1}{\left(z-z^{\prime}\right)^{2-\alpha^{2} / k}}\left[I+\left(z-z^{\prime}\right)^{2} T_{\alpha}^{(\mathrm{pf})}\left(z^{\prime}\right)+\cdots\right], \\
& \psi_{\alpha}(z) \psi_{\beta}\left(z^{\prime}\right)=\frac{K(\alpha, \beta)}{\left(z-z^{\prime}\right)^{1+\alpha \cdot \beta / k}} \psi_{\alpha+\beta}\left(z^{\prime}\right)+\cdots,
\end{aligned}
$$


where

$$
K(\alpha, \beta) \epsilon(\alpha, \beta)=N_{\alpha, \beta} \sqrt{\frac{\alpha^{2} \beta^{2}}{2 k(\alpha+\beta)^{2}}} .
$$

One can assume without loss of generality $\epsilon(\alpha, 0)=\epsilon(0, \alpha)=\epsilon(\alpha,-\alpha)=1$ so that $\epsilon(\alpha, \beta)=$ $\epsilon(-\beta,-\alpha)$.

Substituting (2.4) in (2.2), we find that

$$
\begin{aligned}
& T_{\text {Sug }}(z)=-\frac{1}{2} \cdot \partial \varphi(z) \cdot \partial \varphi(z):+T^{(\mathrm{pf})}(z), \\
& T^{(\mathrm{pf})}(z):=\frac{k}{k+g} \sum_{\alpha \in \Delta_{+}} T_{\alpha}^{(\mathrm{pf})}(z)
\end{aligned}
$$

where we used

$$
\sum_{\alpha \in \Delta_{+}}(\alpha)_{a}(\alpha)_{b}=g \delta_{a b}
$$

and $T_{\alpha}^{(\mathrm{pf})}(z)=T_{-\alpha}^{(\mathrm{pf})}(z)$.

We now wish to recall the interacting boson representation of parafermions [13 as a preparation for later use. For simplicity, assume $\mathbf{g}$ to be simply-laced 1 and let $\chi_{a}^{b}(z)(a=$ $1, \ldots, n, b=1, \ldots, k-1)$ be free bosons normalized by $\left\langle\chi_{a}^{b}(z) \chi_{a^{\prime}}^{b^{\prime}}\left(z^{\prime}\right)\right\rangle=-\delta_{a a^{\prime}} \delta_{b b^{\prime}} \log (z-$ $\left.z^{\prime}\right)$. Let $\lambda_{1}, \ldots, \lambda_{k}$ be the weights in the vector representation of $s u(k)$ which can be considered as $(k-1)$-dimensional vectors satisfying

$$
\sum_{j=1}^{k} \lambda_{j}=0, \quad \lambda_{i} \cdot \lambda_{j}=\delta_{i j}-\frac{1}{k}, \quad \sum_{i=1}^{k}\left(\lambda_{i}\right)_{a}\left(\lambda_{i}\right)_{b}=\delta_{a b}
$$

Notice that $\Delta(s u(k))=\left\{\lambda_{i}-\lambda_{j} \mid i \neq j\right\}$ where $\Delta(s u(k))$ is the set of roots of $s u(k)$. Then the parafermions can be represented by 13

$$
\psi_{\alpha}(z) c_{\alpha}=\frac{1}{\sqrt{k}} \sum_{j=1}^{k} \cdot \exp \left[i \alpha \otimes \lambda_{j} \cdot \chi(z)\right]_{.} c_{\alpha}^{j}
$$

1 The Dynkin diagram of any non simply-laced Lie algebra can be obtained by folding that of an appropriate simply-laced Lie algebra. Using this fact, one can find [14] a vertex operator construction for the level 1 representation of the affine Lie algebra associated with an arbitrary non simply-laced Lie algebra. Hence there is no serious obstacle in extending the results in [13] for a non simply-laced $\mathbf{g}$. 
where $c_{\alpha}^{j}$ 's are $k$ copies of the cocycle factors of $\mathbf{g}$. In this representation,

$$
T_{\alpha}^{(\mathrm{pf})}(z)=\frac{1}{k}\left[-\frac{1}{2} \cdot(\alpha \cdot \partial \chi(z)) \cdot(\alpha \cdot \partial \chi(z))^{*}+\sum_{\beta \in \Delta(\operatorname{su}(k))} \cdot \exp [i \alpha \otimes \beta \cdot \chi(z)]^{*} c_{\alpha}^{\beta}\right]
$$

and hence

$$
T^{(\mathrm{pf})}(z)=\frac{1}{k+g}\left[-\frac{g}{2}: \partial \chi(z) \cdot \partial \chi(z)^{*}+\sum_{\alpha \in \Delta_{+}} \sum_{\beta \in \Delta(s u(k))}: \exp [i \alpha \otimes \beta \cdot \chi(z)]^{*} c_{\alpha}^{\beta}\right],
$$

where $c_{\alpha}^{\beta}=c_{\alpha}^{i} c_{-\alpha}^{j}$ for $\beta=\lambda_{i}-\lambda_{j}$.

Let $P_{+}^{k}=\left\{\Lambda \in \sum_{i=1}^{n} \mathbf{Z}_{\geq 0} \omega_{i}: \Lambda \cdot \theta \leq k\right\}$. The primary fields of the WZW model are given by

$$
\left(\Phi_{\mathrm{WZW}}\right)_{\lambda}^{\Lambda}(z)=\phi_{\lambda}^{\Lambda}(z)^{*} \cdot \exp [i \lambda \cdot \varphi(z) / \sqrt{k}] \cdot, \quad \Lambda \in P_{+}^{k},
$$

where $\lambda$ is a weight in the highest weight representation of $\mathbf{g}$ with highest weight $\Lambda$ and $\phi_{\lambda}^{\Lambda}(z)$ is a parafermionic field of conformal weight

$$
\frac{\Lambda \cdot(\Lambda+2 \rho)}{2(k+g)}-\frac{\lambda^{2}}{2 k}
$$

The field $\phi_{\lambda}^{\Lambda}(z)$ can be obtained from the parafermionic primary field $\phi_{\Lambda}^{\Lambda}(z)$ by repeatedly taking OPEs with $\psi_{-\alpha}(z), \alpha \in \Delta_{+}$. The conformal weight of $\left(\Phi_{\mathrm{WZW}}\right)_{\lambda}^{\Lambda}(z)$ is $h_{\Lambda}=$ $\frac{\Lambda \cdot(\Lambda+2 \rho)}{2(k+g)}$.

We now comment on the notion of 'charge conjugation' in the WZW model. It is convenient to put the theory on a torus. The $\mathcal{P} \mathcal{T}$ transformation on a torus is given by $S^{2}$ where $S$ is the modular transformation $\tau \rightarrow-1 / \tau$ (since $S^{2}$ reverses the orientations of the two homologically non-trivial cycles on the torus.) Consequently, by $\mathcal{C P} \mathcal{T}$ invariance, $S^{2}=\mathcal{C}$ where $\mathcal{C}$ is the charge conjugation transformation satisfying $\mathcal{C}^{2}=1$. Let $W$ be the Weyl group of $\mathbf{g}$ and let $\ell(w)$ denote the length of $w$ for each $w \in W$. There exists a unique longest element $w_{0}$ in $W$ satisfying 15

$$
\begin{aligned}
& \ell\left(w_{0}\right)=\left|\Delta_{+}\right|, \quad w_{0}^{2}=\mathrm{id}, \\
& \ell\left(w w_{0}\right)=\ell\left(w_{0} w\right)=\ell\left(w_{0}\right)-\ell(w) \quad \text { for all } w \in W, \\
& w_{0}\left(\alpha_{i}\right)=-\alpha_{p(i)}, \quad(i=1, \ldots, n) \quad \text { for some permutation } p, \\
& w_{0}(\theta)=-\theta, \quad w_{0}(\rho)=-\rho .
\end{aligned}
$$


The conformal families of the WZW model are labeled by $P_{+}^{k}$ and the conformal blocks on a torus are the Weyl-Kac characters $\chi_{\Lambda}(\tau), \Lambda \in P_{+}^{k}$. The modular transformation $S$ is given by [16]

$$
(S)_{\Lambda, \Lambda^{\prime}}=(i)^{\left|\Delta_{+}\right|}|P /(k+g) M|^{-1 / 2} \sum_{w \in W}(-1)^{\ell(w)} e^{-\frac{2 \pi i}{k+g} w(\Lambda+\rho) \cdot\left(\Lambda^{\prime}+\rho\right)} .
$$

Noting that $S$ is a symmetric and unitary matrix, it follows that $S^{*}=S \mathcal{C}=\mathcal{C} S$. Using this and 2.17$)$ it is easy to see that

$$
(\mathcal{C})_{\Lambda, \Lambda^{\prime}}=\delta_{\bar{\Lambda}, \Lambda^{\prime}}
$$

where $\bar{\Lambda}=-w_{0}(\Lambda)$ is the highest weight in the complex conjugate representation of the irreducible representation of $\mathbf{g}$ with highest weight $\Lambda$. Note that $\Lambda \in P_{+}^{k} \Leftrightarrow \bar{\Lambda} \in P_{+}^{k}$ and $h_{\bar{\Lambda}}=h_{\Lambda}$ by virtue of $(2.17)$. Therefore the field conjugate to $\left(\Phi_{\mathrm{WZW}}\right)_{\lambda}^{\Lambda}(z)$ is given by $\left(\Phi_{\mathrm{WZW}}\right)_{-\lambda}^{\bar{\Lambda}}(z)$ satisfying

$$
\left\langle\left(\Phi_{\mathrm{WZW}}\right)_{\lambda}^{\Lambda}(z)\left(\Phi_{\mathrm{WZW}}\right)_{-\lambda}^{\bar{\Lambda}}\left(z^{\prime}\right)\right\rangle=\frac{1}{\left(z-z^{\prime}\right)^{2 h_{\Lambda}}}
$$

Before closing this section, we should mention some auxiliary facts to be used in the next section. Notice that

$$
E_{-\alpha}(z) E_{\alpha}(z):=T_{\alpha}^{(\mathrm{pf})}(z)-\frac{1}{2 k}:(\alpha \cdot \partial \varphi(z))^{2}:-\frac{1}{2 \sqrt{k}} i \alpha \cdot \partial^{2} \varphi(z) .
$$

Define $[X]_{m}$ for any field $X(z)$ of conformal weight $\Delta_{X}$ and for each $m \in \mathbf{Z}$ by $X(z)=$ $\sum_{m \in \mathbf{Z}}[X]_{m} z^{-m-\Delta_{X}}$. From $\left[E_{\alpha}\right]_{m}\left(\Phi_{\mathrm{WZW}}\right)_{\Lambda}^{\Lambda}(0)|0\rangle=0, m \geq 0, \alpha \in \Delta_{+}$, we observe that

$$
\left[E_{-\alpha} E_{\alpha}^{*}\right]_{0}\left(\Phi_{\mathrm{WZW}}\right)_{\Lambda}^{\Lambda}(0)|0\rangle=0, \quad \alpha \in \Delta_{+} \cdot
$$

Then it follows from (2.21) and (2.22) that

$$
\left[T_{\alpha}^{(\mathrm{pf})}\right]_{0} \phi_{\Lambda}^{\Lambda}(0)|0\rangle=\left(\frac{1}{2 k} \alpha \cdot \Lambda-\frac{1}{2 k^{2}}(\alpha \cdot \Lambda)^{2}\right) \phi_{\Lambda}^{\Lambda}(0)|0\rangle, \quad \alpha \in \Delta_{+} .
$$

As a consistency check we find

$$
\begin{aligned}
{\left[T^{(\mathrm{pf})}\right]_{0} \phi_{\Lambda}^{\Lambda}(0)|0\rangle } & =\frac{k}{k+g} \sum_{\alpha \in \Delta_{+}}\left(\frac{1}{2 k} \alpha \cdot \Lambda-\frac{1}{2 k^{2}}(\alpha \cdot \Lambda)^{2}\right) \phi_{\Lambda}^{\Lambda}(0)|0\rangle \\
& =\left(\frac{\Lambda \cdot(\Lambda+2 \rho)}{2(k+g)}-\frac{\Lambda^{2}}{2 k}\right) \phi_{\Lambda}^{\Lambda}(0)|0\rangle .
\end{aligned}
$$




\section{Higher-Rank Supersymmetric Models}

We first recall the basic features of the higher-rank supersymmetric models [2, 3]. The model consists of free bosons and parafermions in much the same way as in the WZW model. The difference arises from a particular choice of the Coulomb gas parameters. We set them as follows:

$$
\alpha_{+}=\sqrt{\frac{k+g}{k g}}, \quad \alpha_{+} \alpha_{-}=-\frac{1}{k}, \quad \alpha_{0}=\alpha_{+}+\alpha_{-} .
$$

The stress-energy tensor of the model is

$$
\begin{aligned}
T(z) & =-\frac{1}{2} \cdot(\partial \varphi(z))^{2}:+T^{(\mathrm{pf})}(z) \\
& =\sum_{\alpha \in \Delta_{+}} T_{\alpha}(z)=\sum_{m \in \mathbf{Z}} z^{-m-2} L_{m},
\end{aligned}
$$

where

$$
T_{\alpha}(z)=\frac{c}{d}\left[\frac{1}{2}:\left(i \alpha_{+} \alpha \cdot \partial \varphi(z)\right)^{2} \cdot+T_{\alpha}^{(\mathrm{pf})}(z)\right]=\sum_{m \in \mathbf{Z}} z^{-m-2} L_{m}^{\alpha},
$$

and $T(z)$ satisfies the Virasoro algebra with the same central charge as the WZW model, namely, the value $c$ given by (2.3). The fundamental fields in the model are

$$
\Psi_{\nu}^{\Lambda, \mu}(z)=\phi_{\nu-\mu}^{\Lambda}(z)^{-} \exp \left[-i\left(\alpha_{+} \mu+\alpha_{-} \nu\right) \cdot \varphi(z)\right]_{.}^{-}, \quad \Lambda \in P_{+}^{k}
$$

where $\mu \in P / g M$ and $\nu \in P /(k+g) M$ are such that $\nu-\mu$ is a weight in the irreducible representation of $\mathbf{g}$ with highest weight $\Lambda$. The conformal weight of $\Psi_{\nu}^{\Lambda, \mu}(z)$ is

$$
\begin{array}{r}
\frac{\Lambda \cdot(\Lambda+2 \rho)}{2(k+g)}-\frac{(\nu-\mu)^{2}}{2 k}+\frac{1}{2}\left(\alpha_{+} \mu+\alpha_{-} \nu\right)^{2} \\
=\frac{\Lambda \cdot(\Lambda+2 \rho)-\nu^{2}}{2(k+g)}+\frac{\mu^{2}}{2 g} .
\end{array}
$$

The higher-rank supercurrents are defined by

$$
G^{\alpha}(z)=\sqrt{\frac{c}{d}} \psi_{\alpha}(z)^{*} e^{i \alpha_{+} \alpha \cdot \varphi(z)} \cdot, \quad \alpha \in \Delta .
$$

The conformal weight of $G^{\alpha}(z)$ is $\Delta_{\alpha}=1+\frac{\alpha^{2}}{2 g}$ and, in the case $\mathbf{g}=s u(2), G^{\alpha}$ 's reduce to the two supercurrents of the $N=2$ minimal model. We also consider the $u(1)^{n}$ currents defined by

$$
J^{a}(z)=\frac{i}{\alpha_{+}} \partial \varphi_{a}(z)=\sum_{n \in \mathbf{Z}} z^{-n-1} J_{n}^{a}, \quad a=1, \ldots, n
$$


The operators $G^{\alpha}, J$ and $T_{\alpha}$ are the basic generators of the chiral algebra and since parafermions can be represented by a set of bosons as mentioned in the previous section, one can investigate the structure of the chiral algebra without ambiguity starting from $G^{\alpha}$, $J$ and $T_{\alpha}$. It turns out that our chiral algebra is non-local as well as non-linear. Here we merely quote the OPEs we need in what follows:

$$
\begin{aligned}
G^{\alpha}(z) G^{\beta}\left(z^{\prime}\right) & =\frac{\sqrt{\frac{c}{d}} K(\alpha, \beta)}{\left(z-z^{\prime}\right)^{1-\alpha \cdot \beta / g}}\left[G^{\alpha+\beta}\left(z^{\prime}\right)+\cdots\right] \\
G^{\alpha}(z) G^{-\alpha}\left(z^{\prime}\right) & =\frac{\frac{c}{d}}{\left(z-z^{\prime}\right)^{2 \Delta_{\alpha}}}+\frac{\frac{1}{g} \alpha \cdot J\left(z^{\prime}\right)}{\left(z-z^{\prime}\right)^{2 \Delta_{\alpha}-1}} \\
& +\frac{1}{\left(z-z^{\prime}\right)^{2 \Delta_{\alpha}-2}}\left[T_{\alpha}\left(z^{\prime}\right)+\frac{1}{2 g} \alpha \cdot \partial J\left(z^{\prime}\right)+\cdots\right] .
\end{aligned}
$$

The explicit expressions (3.3), (3.6) and (3.7) imply the existence of automorphism in the algebra:

$$
\begin{aligned}
& T_{\alpha}(z) \rightarrow T_{\alpha}(z)+\frac{i}{g}\left(\alpha \cdot \frac{d \beta(z)}{d z}\right)(\alpha \cdot J(z))-\frac{c}{2 d}\left(\alpha \cdot \frac{d \beta(z)}{d z}\right)^{2} \\
& J(z) \rightarrow J(z)+i \frac{c g}{d} \frac{d \beta(z)}{d z} \\
& G^{\alpha}(z) \rightarrow e^{i \alpha \cdot \beta(z)} G^{\alpha}(z)
\end{aligned}
$$

generated by the $u(1)^{n}$ gauge transformation

$$
\varphi(z) \rightarrow \varphi(z)+\frac{1}{\alpha_{+}} \beta(z),
$$

where $\beta(z)$ is an $n$-dimensional vector whose components are arbitrary analytic functions. Setting in particular $\beta(z)=i \bar{\eta} \log z$ in (3.9) with $\bar{\eta}$ being an $n$-dimensional vector we have

$$
\begin{aligned}
L_{m}^{\alpha} & \rightarrow L_{m}^{\alpha}-\frac{1}{g}(\alpha \cdot \bar{\eta})\left(\alpha \cdot J_{m}\right)+\frac{c}{2 d}(\alpha \cdot \bar{\eta})^{2} \delta_{m, 0}, \\
J_{m}^{a} & \rightarrow J_{m}^{a}-\frac{c g}{d} \bar{\eta}^{a} \delta_{m, 0}, \\
G_{r}^{\alpha} & \rightarrow G_{r+\alpha \cdot \bar{\eta}}^{\alpha}
\end{aligned}
$$

where

$$
G^{\alpha}(z)=\sum_{r} z^{-r-\Delta_{\alpha}} G_{r}^{\alpha} .
$$

This is a natural generalization of the one-parameter spectral flow in $N=2$ superconformal algebra [17] to the $n$-parameter flow in the higher-rank case. 


\subsection{The NS sector and Chiral Primary Fields}

We next seek for the analogue of the chirality condition and chiral primary fields in $N=2$ superconformal field theory [18]. For this purpose let us first define the fields in the NS sector as the set of fields $A_{N S}(z)$ which are local with respect to $G^{\alpha}(z)$ :

$$
G^{\alpha}(z) A_{N S}(0)=\sum_{r \in \mathbf{Z}-\Delta_{\alpha}} z^{-r-\Delta_{\alpha}}\left(G_{r}^{\alpha} A_{N S}\right)(0)
$$

If a state $\left|\Phi_{p}\right\rangle=\Phi_{p}(0)|0\rangle$, where $\Phi_{p}(z)$ is a field in the NS sector, is annihilated by all the positive-mode generators $L_{m}, J_{m}^{a}$ and $G_{m-\frac{\alpha^{2}}{2 g}}^{\alpha}$ with $m>0$, then it is called a primary state. A primary state $\left|\Phi_{p}\right\rangle$ is labeled by the eigenvalues of $L_{0}$ and $J_{0}^{a}$

$$
L_{0}\left|\Phi_{p}\right\rangle=h\left|\Phi_{p}\right\rangle, \quad J_{0}^{a}\left|\Phi_{p}\right\rangle=q_{a}\left|\Phi_{p}\right\rangle, \quad a=1, \ldots, n
$$

We also define a chiral state $\left|\Phi_{c}\right\rangle$ in the NS sector by requiring

$$
G_{-\frac{\alpha_{i} 2}{2 g}}^{\alpha_{i}}\left|\Phi_{c}\right\rangle=0, \quad i=1, \ldots, n
$$

where we recall $\alpha_{1}, \ldots, \alpha_{n}$ are the simple roots of $\mathbf{g}$.

If a chiral state also satisfies the primary state conditions it is called a chiral primary state. Actually we have

$$
G_{l-\frac{\alpha^{2}}{2 g}}^{\alpha}\left|\Phi_{c \wedge p}\right\rangle=0, \quad l \geq 0, \quad \alpha \in \Delta_{+}
$$

for any chiral primary state $\left|\Phi_{c \wedge p}\right\rangle$. This can be seen as follows. First it is clear that (3.16) is satisfied when $\alpha$ is a simple root. By resorting to the standard procedure [10,19] one can deduce from (3.8a) that in the NS sector

$$
\begin{gathered}
\sum_{l=0}^{\infty} C_{l}^{(-\alpha \cdot \beta / g)}\left(G_{p-l-\frac{\alpha \cdot(\alpha+2 \beta)}{2 g}}^{\alpha} G_{q+l-\frac{\beta^{2}}{2 g}}^{\beta}+G_{q-l-\frac{\beta \cdot(\beta+2 \alpha)}{2 g}}^{\beta} G_{p+l-\frac{\alpha^{2}}{2 g}}^{\alpha}\right) \\
=\sqrt{\frac{c}{d}} K(\alpha, \beta) G_{p+q-\frac{(\alpha+\beta)^{2}}{2 g}}^{\alpha+\beta}
\end{gathered}
$$

where $p, q \in \mathbf{Z}$ and

$$
C_{l}^{(a)}=(-1)^{l} \frac{\Gamma(a+1)}{l ! \Gamma(a+1-l)} .
$$


Suppose that (3.16) is true for two positive roots $\alpha$ and $\beta$ such that $\alpha+\beta$ is again a positive root. Then having both sides of (3.17) with $p=q=0$ act on the chiral primary state $\left|\Phi_{c \wedge p}\right\rangle$ we find

$$
G_{-\frac{(\alpha+\beta)^{2}}{2 g}}^{\alpha+\beta}\left|\Phi_{c \wedge p}\right\rangle=0 .
$$

Hence taking account of the primary conditions, (3.16) is also true for the positive root $\alpha+\beta$. This completes the proof.

It also follows from (3.88) that in the NS sector

$$
\begin{aligned}
\sum_{l=0}^{\infty} C_{l}^{\left(\alpha^{2} / g-1\right)} & \left(G_{m-l+\frac{\alpha^{2}}{2 g}}^{-\alpha} G_{m^{\prime}+l-\frac{\alpha^{2}}{2 g}}^{\alpha}+G_{m^{\prime}-1-l+\frac{\alpha^{2}}{2 g}}^{\alpha} G_{m+1+l-\frac{\alpha^{2}}{2 g}}^{-\alpha}\right) \\
& =\left[\frac{c}{2 d} m(m+1) \delta_{m+m^{\prime}, 0}+L_{m+m^{\prime}}^{\alpha}-\frac{1}{2 g}\left(m-m^{\prime}+1\right) \alpha \cdot J_{m+m^{\prime}}\right],
\end{aligned}
$$

where $m, m^{\prime} \in \mathbf{Z}$. Let us consider a secondary state $G_{-\frac{\alpha^{2}}{2 g}}^{\alpha}\left|\Phi_{p}\right\rangle$ for a NS primary state $\left|\Phi_{p}\right\rangle$ and $\alpha \in \Delta_{+}$. Define the adjoint of $G_{r}^{\alpha}$ by $\left(G_{r}^{\alpha}\right)^{\dagger}=G_{-r}^{-\alpha}$. Using (3.20) we evaluate the norm of this secondary state. It is then immediate to find a bound imposed by unitarity

$$
h_{\alpha} \geq \frac{1}{2 g} \alpha \cdot q,
$$

where $L_{0}^{\alpha}\left|\Phi_{p}\right\rangle=h_{\alpha}\left|\Phi_{p}\right\rangle$. For a chiral primary state this bound is saturated and hence there holds a linear relation between its conformal weight $h$ and $u(1)^{n}$ charge $q$ :

$$
h=\frac{1}{g} \rho \cdot q,
$$

Having $G_{-1-\frac{\alpha^{2}}{2 g}}^{\alpha}$ with $\alpha \in \Delta_{+}$act on a chiral primary state and evaluating its norm we get a unitarity bound

$$
\frac{1}{g} \alpha \cdot q \leq \frac{c}{d}, \quad \alpha \in \Delta_{+},
$$

and hence we find the restriction

$$
0 \leq h \leq \frac{d-n}{4 d} c,
$$

for chiral primary states. As a result the number of chiral primary fields is finite as long as the theory is non-degenerate. 
The explicit form of chiral primary fields is obtained as

$$
\left\{\Phi_{\Lambda}(z)=\Psi_{\Lambda}^{\Lambda, 0}(z) \mid \Lambda \in P_{+}^{k}\right\} .
$$

Using (3.20) and (2.23) it is easy to check that the conditions (3.16) are fulfilled for $\left|\Phi_{\Lambda}\right\rangle$ and the relation $(3.22)$ is satisfied:

$$
h_{\Lambda}=\frac{1}{g} \rho \cdot q_{\Lambda} \quad \text { with } \quad q_{\Lambda}=\frac{g}{k+g} \Lambda .
$$

Then following the standard argument we find that there are no short-distance singularities between two chiral primary fields

$$
\Phi_{\Lambda}(z) \Phi_{\Lambda^{\prime}}\left(z^{\prime}\right) \sim \Phi_{\Lambda+\Lambda^{\prime}}\left(z^{\prime}\right),
$$

where we should understand that $\Phi_{\Lambda+\Lambda^{\prime}}(z)=0$ if $\Lambda+\Lambda^{\prime} \notin P_{+}^{k}$. Hence a finite nilpotent ring is generated by our chiral primary fields. We will refer to this ring as chiral ring.

One can more generically consider a set of conditions

$$
G_{-\frac{\alpha_{i}}{2 g}}^{w\left(\alpha_{i}\right)}|\Phi\rangle=0, \quad i=1, \ldots, n,
$$

for each $w \in W$ instead of (3.15). Denote as $\mathcal{R}_{w}$ the set of primary fields in the NS sector obeying (3.28). Then we have

$$
\mathcal{R}_{w}=\left\{\Psi_{w(\Lambda)}^{\Lambda, 0} \mid \Lambda \in P_{+}^{k}\right\} .
$$

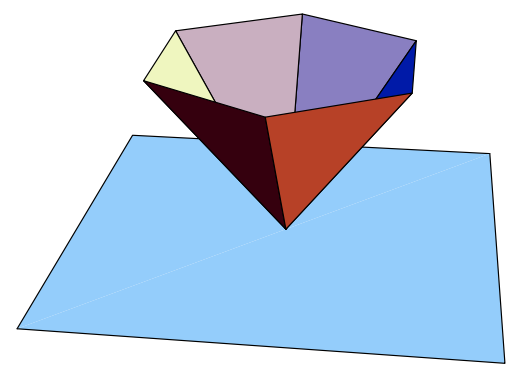

Figure 1. The distribution of $(h, q)$ for $\underset{w \in W}{\cup} \mathcal{R}_{w}$ in the case $\mathbf{g}=s u(3)$. The $h$-axis is chosen to be vertical. For easy reference the $h=0$ plane is also depicted. 
Clearly, in this notation $\mathcal{R}_{\text {id }}$ is the chiral ring mentioned above. As in the WZW model, the charge conjugate field of $\Psi_{w(\Lambda)}^{\Lambda, 0} \in \mathcal{R}_{w}$ is $\Psi_{w w_{0}(\bar{\Lambda})}^{\bar{\Lambda}, 0} \in \mathcal{R}_{w w_{0}}$. Therefore, in view of the property $\Lambda \in P_{+}^{k} \Leftrightarrow \bar{\Lambda} \in P_{+}^{k}$, the conjugate set of $\mathcal{R}_{w}$ coincides with $\mathcal{R}_{w w_{0}}$. Each $\mathcal{R}_{w}$, $w \in W$ becomes a ring isomorphic to the chiral ring $\mathcal{R}_{\text {id }}$. However it should be noted that although the set $\underset{w \in W}{\cup} \mathcal{R}_{w}$ is apparently conjugation symmetric, in general two fields $\phi \in \mathcal{R}_{w}$ and $\phi^{\prime} \in \mathcal{R}_{w^{\prime}}$ with $w \neq w^{\prime}$ are not mutually local and hence we cannot associate a good algebraic structure with the whole set $\underset{w \in W}{\cup} \mathcal{R}_{w}$.

\subsection{The $R$ sector}

Specializing the spectral flow parameter $\bar{\eta}=\frac{\eta}{g} \rho$ with $\eta \in \mathbf{R}$ in (3.9) we obtain a one-parameter flow

$$
\begin{aligned}
L_{m}^{\alpha} & \rightarrow L_{m}^{\alpha}-\eta \frac{\alpha \cdot \rho}{g^{2}}\left(\alpha \cdot J_{m}\right)+\eta^{2} \frac{c}{2 d}\left(\frac{\alpha \cdot \rho}{g}\right)^{2} \delta_{m, 0}, \\
J_{m}^{a} & \rightarrow J_{m}^{a}-\eta \frac{c}{d}(\rho)_{a} \delta_{m, 0}, \\
G_{r}^{\alpha} & \rightarrow G_{r+\eta \frac{\alpha \cdot \rho}{g}}^{\alpha},
\end{aligned}
$$

and hence

$$
L_{m} \rightarrow L_{m}-\eta \frac{1}{g} \rho \cdot J_{m}+\eta^{2} \frac{c}{24} \delta_{m, 0} .
$$

In the sector obtained by applying this flow with $\eta=1$ to the NS sector the generators $G_{r}^{\alpha_{1}}, \ldots, G_{r}^{\alpha_{n}}$ and $G_{r}^{-\theta}$ have integer modings. Thus this is a sector analogous to one of the R sectors in $N=2$ superconformal theory. The chirality conditions (3.15) as well as the primary state conditions for $G_{r}^{-\theta}$ are converted into ground-state conditions

$$
G_{r}^{\alpha_{i}}|R\rangle=0, \quad G_{r}^{-\theta}|R\rangle=0, \quad r=0,1,2, \ldots
$$

It is clear that under this flow a chiral primary field $\Phi_{\Lambda}=\Psi_{\Lambda}^{\Lambda, 0}$ turns into a Ramond ground field $\Psi_{\Lambda+\rho}^{\Lambda, \rho}$. More generally, under the flow corresponding to the choice $\bar{\eta}=\frac{1}{g} w(\rho)$, $w \in W$ in (3.30) the set $\mathcal{R}_{w}$ flows to the set

$$
\mathcal{V}_{w}=\left\{\Psi_{w(\Lambda+\rho)}^{\Lambda, w(\rho)}(z) \mid \Lambda \in P_{+}^{k}\right\}
$$

by the rule $\Psi_{w(\Lambda)}^{\Lambda, 0} \rightarrow \Psi_{w(\Lambda+\rho)}^{\Lambda, w(\rho)}$. An element $\Psi_{w(\Lambda+\rho)}^{\Lambda, w(\rho)}$ of $\mathcal{V}_{w}$ has quantum numbers

$$
h=\frac{c}{24}, \quad q=\frac{g}{k+g} w(\Lambda+\rho)-w(\rho) .
$$




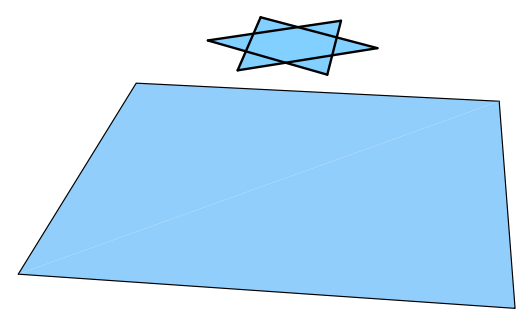

Figure 2. The distribution of $(h, q)$ for $\underset{w \in W}{\cup} \mathcal{V}_{w}$ in the case $\mathbf{g}=s u(3)$ and the $h=0$ plane. 'Star of David' sits at the height of $c / 24$.

This spectrum can also be obtained by an analysis of branching relations [2].

The charge conjugate field of $\Psi_{w(\Lambda+\rho)}^{\Lambda, w(\rho)} \in \mathcal{V}_{w}$ is $\Psi_{w w_{0}(\bar{\Lambda}+\rho)}^{\bar{\Lambda}, w w_{0}(\rho)} \in \mathcal{V}_{w w_{0}}$ and hence the conjugate set of $\mathcal{V}_{w}$ is $\mathcal{V}_{w w_{0}}$. Therefore the whole set $\underset{w \in W}{\cup} \mathcal{V}_{w}$ of $\mathrm{R}$ ground fields is symmetric under charge conjugation.

At this juncture we should comment on the special feature of the case $\mathbf{g}=s u(2)$, i.e. the $N=2$ minimal model. In this case we have $W=\{ \pm 1\}$ and $\mathcal{V}_{w=+1}=\mathcal{V}_{w=-1}$. The latter follows from

$$
\begin{aligned}
& \mathcal{V}_{w=-1} \ni \phi_{-l}^{l}(z)^{*} \exp \left[+i\left\{\alpha_{+}+\alpha_{-}(l+1)\right\} \varphi(z) / \sqrt{2}\right] \\
&=\phi_{k-l}^{k-l}(z)^{*} \cdot \exp \left[-i\left\{\alpha_{+}+\alpha_{-}(k-l+1)\right\} \varphi(z) / \sqrt{2}\right]: \in \mathcal{V}_{w=+1}
\end{aligned}
$$

where we wrote $\phi_{m}^{l}(z)$ instead of $\phi_{m / \sqrt{2}}^{l / \sqrt{2}}(z)$ following the standard convention. In deriving (3.35) we used $g \alpha_{+}=-(k+g) \alpha_{-}$and the symmetry property $\phi_{-l}^{l}(z)=\phi_{k-l}^{k-l}(z)$ which is the consequence of diagram automorphism. Therefore in contrast to the generic case $\mathrm{g} \neq s u(2)$ where $\mathcal{V}_{w=\text { id }}$ alone is certainly not charge-conjugation symmetric, in the case $\mathrm{g}=s u(2)$ the set $\mathcal{V}_{w=+1}$ is the charge-conjugation symmetric set of the Ramond ground fields. This in turn implies that the set of chiral primary fields $\mathcal{R}_{w=+1}$, which is mapped onto $\mathcal{V}_{w=+1}$ by a spectral flow, also has a symmetry. This is nothing but the Poincaré duality of the chiral ring. We will say more about this in the next section. Recall that 


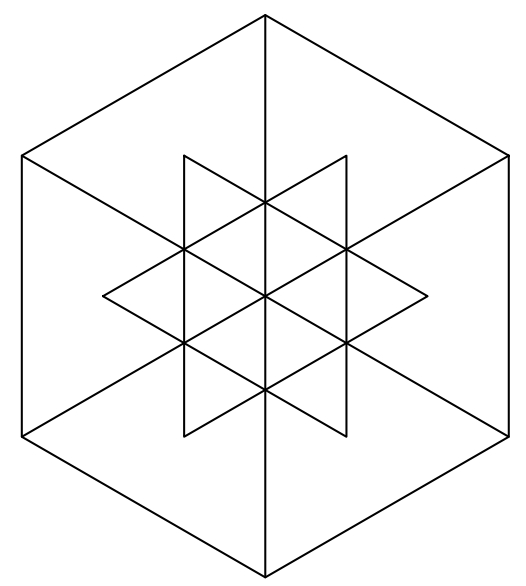

Figure 3. The $u(1)^{n}$ charge distribution of both $\underset{w \in W}{\cup} \mathcal{R}_{w}$ and $\underset{w \in W}{\cup} \mathcal{V}_{w}$ for $\mathbf{g}=s u(3)$. The six outer equilateral triangles forming a hexagon correspond to $\underset{w \in W}{\cup} \mathcal{R}_{w}$ while the two inside ones which are overlaid correspond to $\underset{w \in W}{\cup} \mathcal{V}_{w}$. Each outer triangle goes to one of the two inside ones by a spectral flow mentioned in the text.

it is quite generally argued in $N=2$ superconformal field theory [18 that $u(1)$ chargeconjugation symmetry of the Ramond vacua translates into the Poincaré duality of the chiral ring.

\section{Topological Conformal Field Theory}

As we have seen in the previous section the higher-rank supersymmetry turns out to be a Lie algebraic extension of $N=2$ superconformal symmetry. According to general arguments [4, [5] every $N=2$ theory yields a topological field theory. So a natural problem arises of whether we can produce a sort of topological field theories starting from our higher-rank supersymmetric models. Answering this is the main theme of this section. We will argue that it is possible to construct topological field theories but to render them sensible we must adopt some special procedures. 
As in the twisted $N=2$ theory we begin by assuming that the new stress-energy tensor is given by

$$
\begin{aligned}
\hat{T}(z) & =T(z)+\frac{1}{g} \rho \cdot \partial J(z) \\
& =-\frac{1}{2} \cdot \partial \varphi(z) \cdot \partial \varphi(z)^{*}+i \alpha_{0} \rho \cdot \partial^{2} \varphi(z)+T^{(\mathrm{pf})}(z)
\end{aligned}
$$

which generates the Virasoro algebra with a vanishing central charge. In the usual twisted $N=2$ theory the twisted stress-energy tensor can be expressed as

$$
\hat{T}_{N=2}(z)=\oint_{z} d z^{\prime} G^{+}\left(z^{\prime}\right) G^{-}(z)
$$

and is claimed to be BRST-exact by interpreting $\oint G^{+}$as the BRST charge. We want a similar 'BRST-exact' 2 expression for $\hat{T}(z)$. To our luck there exists such a formula

$$
\hat{T}(z) \propto T_{s}(z)
$$

with $T_{s}(z)$ given by

$$
\begin{aligned}
T_{s}(z)=\oint \prod_{i=1}^{n} \prod_{p=1}^{a_{i}} d z_{i, p} G^{\alpha_{1}}\left(z_{1,1}\right) \cdots G^{\alpha_{1}}\left(z_{1, a_{1}}\right) \\
\quad \times \cdots \times G^{\alpha_{n}}\left(z_{n, 1}\right) \cdots G^{\alpha_{n}}\left(z_{n, a_{n}}\right) G^{-\theta}(z) \\
\equiv \oint\left(G^{\alpha_{1}}\right)^{a_{1}} \cdots\left(G^{\alpha_{n}}\right)^{a_{n}} G^{-\theta}(z)
\end{aligned}
$$

where the positive integers $a_{1}, \ldots, a_{n}$ are defined via $\theta=\sum_{i=1}^{n} a_{i} \alpha_{i}$ and the contour of the multiple integrals must be chosen appropriately $\mathrm{B}$. Notice that $G^{\alpha_{i}}(z)$ 's have conformal weight 1 with respect to $\hat{T}(z)$ while $G^{-\theta}(z)$ has 2 . The expression (4.3) was first proposed in [7] in the context of $c=0 \mathrm{GKO}$ coset models. We note that the twisted stress-energy tensor (4.1) coincides with that appearing in the $G_{k} \times G_{0} / G_{k}$ model. In what follows we first dwell upon the validity of (4.3) and then we move on to the actual construction of topological field theories.

2 We will explain later what is meant by 'BRST-exact'.

3 Throughout the paper we are rather cavalier about the treatment of multiple integral contours although we do not pretend to have succeeded in handling rigorously the contours. Anyway simpleminded treatment indeed works as we shall see. 


\subsection{BRST-exactness of the Stress-Energy Tensor - Multiple Integrals}

Our goal in this subsection is to perform some sample calculations explicitly verifying the BRST-exactness (4.3) of the twisted stress-energy tensor. It remains as a mathematically interesting (but perhaps pains-taking) task to accomplish these calculations in full generality, namely, to prove (4.3) for all simple Lie algebras with arbitrary levels. We hope we can return to this challenging problem in future.

Before launching to the calculations, we require some definitions; let $M_{i j}$ and $m_{i}$ 's be given by

$$
\begin{aligned}
& M_{i j}=\frac{4}{\alpha_{i}^{2} \alpha_{j}^{2}} \omega_{i} \cdot \omega_{j}, \\
& 2 \rho=\sum_{i=1}^{n} m_{i} \alpha_{i} .
\end{aligned}
$$

In the sequel we will frequently use the relation

$$
\sum_{i, j=1}^{n} M_{i j}\left(\alpha_{i}\right)_{a}\left(\alpha_{j}\right)_{b}=\delta_{a b} .
$$

The necessary Lie algebraic data are summarized in Tables $\mathbf{1 - 3 .}$

- $\mathrm{g}=\mathcal{A}_{n}, k \geq 1$

To evaluate $\hat{T}_{s}(z)$ it is convenient to employ the interacting boson representation of parafermions reviewed in sect.2. After some standard manipulations we find

$$
\begin{aligned}
\hat{T}_{s}(z) & \propto \oint \prod_{i=1}^{n} d z_{i} \\
& \times \sum_{j_{0}, \ldots, j_{n}=1}^{k} \prod_{i=1}^{n-1}\left(z_{i}-z_{i+1}\right)^{-\left(\delta_{j_{i}, j_{i+1}}+\frac{1}{g}\right)}\left(z_{1}-z\right)^{-\left(\delta_{j_{1}, j_{0}}+\frac{1}{g}\right)}\left(z_{n}-z\right)^{-\left(\delta_{j_{n}, j_{0}}+\frac{1}{g}\right)} \\
& \times: \exp \left[i \alpha_{+} \sum_{i=1}^{n} \alpha_{i} \cdot\left(\varphi\left(z_{i}\right)-\varphi(z)\right)\right] \\
& \times: \exp \left[i \sum_{i=1}^{n}\left\{\alpha_{i} \otimes \lambda_{j_{i}} \cdot \chi\left(z_{i}\right)-\alpha_{i} \otimes \lambda_{j_{0}} \cdot \chi(z)\right\}\right]: c_{\alpha_{1}}^{j_{1}} \cdots c_{\alpha_{n}}^{j_{n}} c_{-\theta}^{j_{0}} \\
& \propto \oint \prod_{i=1}^{n} d z_{i}
\end{aligned}
$$




$$
\begin{aligned}
& \times\left[\prod_{i=1}^{n-1}\left(z_{i}-z_{i+1}\right)\left(z_{1}-z\right)\left(z_{n}-z\right)\right]^{-1-\frac{1}{g}}\left[1+\frac{1}{2} \sum_{1 \leq i, j \leq n}\left(z_{i}-z\right)\left(z_{j}-z\right) A_{i j}(z)\right. \\
& +\sum_{i=1}^{n}\left(z_{i}-z\right)^{2} B_{i}(z)+\left(z_{1}-z\right) \sum_{i=1}^{n-1}\left(z_{i}-z_{i+1}\right) \mathcal{T}_{\alpha_{1}+\cdots+\alpha_{i}}(z) \\
& +\left(z_{1}-z\right)\left(z_{n}-z\right) \mathcal{T}_{\alpha_{1}+\cdots+\alpha_{n}}(z)-\sum_{2 \leq i \leq j \leq n-1}\left(z_{i-1}-z_{i}\right)\left(z_{j}-z_{j+1}\right) \mathcal{T}_{\alpha_{i}+\cdots+\alpha_{j}}(z) \\
& \left.-\sum_{2 \leq i \leq n}\left(z_{i-1}-z_{i}\right)\left(z_{n}-z\right) \mathcal{T}_{\alpha_{i}+\cdots+\alpha_{n}}(z)+\text { higher order terms }\right]
\end{aligned}
$$

where

$$
\begin{aligned}
& A_{i j}(z)=:\left(i \alpha_{+} \alpha_{i} \cdot \partial \varphi(z)\right)\left(i \alpha_{+} \alpha_{j} \cdot \partial \varphi(z)\right)^{*}+\frac{1}{k}:\left(i \alpha_{i} \cdot \partial \chi(z)\right) \cdot\left(i \alpha_{j} \cdot \partial \chi(z)\right)_{\cdot}, \\
& B_{i}(z)=\frac{1}{2} i \alpha_{+} \alpha_{i} \cdot \partial^{2} \varphi(z)
\end{aligned}
$$

and

$$
\mathcal{T}_{\alpha}(z)=\frac{1}{k} \sum_{\beta \in \Delta(s u(k))}: \exp [i \alpha \otimes \beta \cdot \chi(z)]^{*} c_{\alpha}^{\beta}, \quad \alpha \in \Delta_{+} .
$$

In deriving (4.7) we have used the properties of cocycle factors summarized in sect.2.

Next we make change of variables $z_{i} \rightarrow z+\prod_{l=1}^{i} u_{l}, i=1, \ldots, n$ and integrate over $u_{1}$ by the Cauchy residue formula to reach

$$
\begin{aligned}
\hat{T}_{s}(z) & \propto \oint \prod_{i=2}^{n} d u_{i} u_{i}^{\left(\frac{i}{g}-1\right)-1}\left(1-u_{i}\right)^{-\frac{1}{g}-1} \\
& \times\left[\frac{1}{2}\left(A_{11}(z)+\sum_{i=1}^{n}\left(u_{2} \cdots u_{i}\right)^{2} A_{i i}(z)\right)+\sum_{i=2}^{n}\left(u_{2} \cdots u_{i}\right) A_{1 i}(z)\right. \\
& +\sum_{2 \leq i<j \leq n}\left(u_{2} \cdots u_{i}\right)\left(u_{2} \cdots u_{j}\right) A_{i j}(z)+B_{1}(z)+\sum_{i=2}^{n}\left(u_{2} \cdots u_{i}\right)^{2} B_{i}(z) \\
& +\left(1-u_{2}\right) \mathcal{T}_{\alpha_{1}}(z)+\sum_{i=2}^{n-1}\left(u_{2} \cdots u_{i}\right)\left(1-u_{i+1}\right) \mathcal{T}_{\alpha_{1}+\cdots+\alpha_{i}}(z) \\
& +\left(u_{2} \cdots u_{n}\right) \mathcal{T}_{\alpha_{1}+\cdots+\alpha_{n}}(z)-u_{2}\left(1-u_{2}\right)\left(1-u_{3}\right) \mathcal{T}_{\alpha_{2}}(z) \\
&
\end{aligned}
$$




$$
\begin{aligned}
& -\sum_{i=3}^{n-1}\left(u_{2} \cdots u_{i-1}\right)^{2} u_{i}\left(1-u_{i}\right)\left(1-u_{i+1}\right) \mathcal{T}_{\alpha_{i}}(z) \\
& -\sum_{i=3}^{n-1} u_{2}\left(1-u_{2}\right) u_{3} \cdots u_{i}\left(1-u_{i+1}\right) \mathcal{T}_{\alpha_{2}+\cdots+\alpha_{i}}(z) \\
& -\sum_{3 \leq i<j \leq n-1}\left(u_{2} \cdots u_{i-1}\right)^{2} u_{i}\left(1-u_{i}\right) u_{i+1} \cdots u_{j}\left(1-u_{j+1}\right) \mathcal{T}_{\alpha_{i}+\cdots+\alpha_{j}}(z) \\
& -u_{2}\left(1-u_{2}\right)\left(u_{3} \cdots u_{n}\right) \mathcal{T}_{\alpha_{2}+\cdots+\alpha_{n}}(z) \\
& \quad \sum_{i=3}^{n-1}\left(u_{2} \cdots u_{i-1}\right)^{2} u_{i}\left(1-u_{i}\right)\left(u_{i+1} \cdots u_{n}\right) \mathcal{T}_{\alpha_{i}+\cdots+\alpha_{n}}(z) \\
& \left.-\left(u_{2} \cdots u_{n-1}\right)^{2} u_{n}\left(1-u_{n}\right) \mathcal{T}_{\alpha_{n}}(z)\right]
\end{aligned}
$$

By repeatedly applying the recursion formulae of the beta functions it is now straightforward to check that

$$
\hat{T}_{s}(z) \propto \frac{g}{2} \sum_{i, j=1}^{n} M_{i j} A_{i j}(z)+\sum_{i=1}^{n} m_{i} B_{i}(z)+\sum_{\alpha \in \Delta_{+}} \mathcal{T}_{\alpha}(z),
$$

which amounts to $(4.3)$ with $T^{(\mathrm{pf})}(z)$ given by 2.14 .

- $\mathbf{g}=\mathcal{B}_{n}, k=1$

In this case the parafermions are given by

$$
\psi_{\alpha}(z)= \begin{cases}\psi(z), & \text { if } \alpha \text { is short } \\ 1, & \text { if } \alpha \text { is long }\end{cases}
$$

where $\psi(z)$ is a Majorana fermion satisfying the OPE

$$
\psi(z) \psi\left(z^{\prime}\right)=\frac{1}{z-z^{\prime}}+\cdots,
$$

Consequently,

$$
T_{\alpha}^{(\mathrm{pf})}(z)= \begin{cases}\dot{-}^{\prime}(z) \psi(z)^{*}, & \text { if } \alpha \text { is short } \\ 0, & \text { if } \alpha \text { is long }\end{cases}
$$

and hence

$$
T^{(\mathrm{pf})}(z)=\frac{1}{1+g} \sum_{\alpha \in \Delta_{+}} T_{\alpha}^{(\mathrm{pf})}(z)=\frac{1}{2} \psi^{\prime}(z) \psi(z)^{\bullet} .
$$


Again it is easy to see

$$
\begin{aligned}
\hat{T}_{s}(z) & \propto \oint d z_{1} \prod_{i=2}^{n} d z_{i, 1} d z_{i, 2} \\
& \times\left[\prod_{p=1}^{2}\left(z_{1}-z_{2, p}\right)^{-1}\left(z_{2, p}-z\right)^{-1} \prod_{i=2}^{n-1} \prod_{p, q=1}^{2}\left(z_{i, p}-z_{i+1, q}\right)^{-1}\left(z_{i, 1}-z_{i, 2}\right)^{2}\right]^{1+\frac{1}{g}} \\
& \times\left(z_{n, 1}-z_{n, 2}\right)^{\frac{1}{g} \cdot} \cdot \exp \left[i \alpha_{+}\left\{\alpha_{1} \cdot\left(\varphi\left(z_{1}\right)-\varphi(z)\right)+\sum_{i=2}^{n} \sum_{p=1}^{2} \alpha_{i} \cdot\left(\varphi\left(z_{i, p}\right)-\varphi(z)\right)\right\}\right] \\
& \times\left[1+\left(z_{n, 1}-z_{n, 2}\right)^{*} \psi\left(z_{n, 1}\right) \psi\left(z_{n, 2}\right)^{*}\right] .
\end{aligned}
$$

Setting

$$
\begin{aligned}
& A_{i j}(z)=:\left(i \alpha_{+} \alpha_{i} \cdot \partial \varphi(z)\right)\left(i \alpha_{+} \alpha_{j} \cdot \partial \varphi(z)\right)_{-}, \\
& B_{i}(z)=\frac{1}{2} i \alpha_{+} \alpha_{i} \cdot \partial^{2} \varphi(z) \\
& C(z)=: \psi^{\prime}(z) \psi(z)_{-}^{*}
\end{aligned}
$$

we obtain

Lemma (4.18). For each $l=3,4, \ldots, n$,

$$
\begin{aligned}
\hat{T}_{s}(z) \propto & \oint \prod_{i=l}^{n} d z_{i, 1} d z_{i, 2} \\
\times & \prod_{p=1}^{2}\left(z_{l, p}\left(1-z_{l, p}\right)\right)^{-1-\frac{1}{g}}\left(z_{l, 1}-z_{l, 2}\right)^{-1+\frac{2}{g} l} P_{l}^{\mathcal{B}}\left(z_{\bullet, \bullet}\right) \\
\times & {\left[\left(\frac{g}{2} \sum_{\substack{1 \leq i, j \leq n \\
\min (i, j) \leq l-1}} M_{i j} A_{i j}(z)+\sum_{i=1}^{l-1} m_{i} B_{i}(z)\right)+2 \sum_{l \leq i, j \leq n} f_{l}\left(\zeta_{i}, \zeta_{j}\right) A_{i j}(z)\right.} \\
& \left.\quad+\sum_{i=l}^{n} \sum_{p=1}^{2} f_{l}\left(z_{i, p}, z_{i, p}\right) B_{i}(z)+d_{l}\left(z_{n, 1}-z_{n, 2}\right)^{2} C(z)\right]
\end{aligned}
$$


where we have set $\zeta_{i}=\frac{1}{2}\left(z_{i, 1}+z_{i, 2}\right)$ for $i=3,4, \ldots, n$ and

$$
\begin{aligned}
P_{l}^{\mathcal{B}}\left(z_{\bullet, \bullet}\right) & = \\
= & \begin{cases}{\left[\prod_{p=1}^{2} z_{l+1, p}^{-1}\left(1-z_{l+1, p}\right)^{-1}\right.} \\
\left.\times \prod_{i=l+1}^{n-1} \prod_{p, q=1}^{2}\left(z_{i, p}-z_{i+1, q}\right)^{-1}\left(z_{i, 1}-z_{i, 2}\right)^{2}\right]^{1+\frac{1}{g}}\left(z_{n, 1}-z_{n, 2}\right)^{\frac{1}{g}}, & 3 \leq l \leq n-2, \\
{\left[\prod_{p=1}^{2} z_{n, p}^{-1}\left(1-z_{n, p}\right)^{-1}\right]^{1+\frac{1}{g}}\left(z_{n, 1}-z_{n, 2}\right)^{\frac{1}{g}},} & l=n-1, \\
1, & l=n .\end{cases}
\end{aligned}
$$

The functions $f_{l}(u, v)$ in (4.19) are defined for $l \geq 3$ by

$$
f_{l}(u, v)=a_{l}(u v+(1-u)(1-v))+b_{l}(u(1-v)+v(1-u))+c_{l},
$$

with

$$
\begin{aligned}
a_{l} & =\frac{1}{2} g(l-1)-(l-2)^{2}, \\
b_{l} & =l-2, \\
c_{l} & =\frac{1}{2}(l-2)(l-3),
\end{aligned}
$$

and

$$
d_{l}=(l-1)(g-2(l-2)) .
$$

Proof. For $3 \leq l \leq n-2$ we prove this by induction on $l$.

(i) $l=3$.

First make change of variables $z_{2,1} \rightarrow z+x, z_{2,2} \rightarrow z+x y, z_{1} \rightarrow z+x z_{1}$ and $z_{i, p} \rightarrow$ $z+x z_{i, p}$ for $i=3, \ldots, n$ and $p=1,2$ on the RHS of (4.16) and then integrate over $x$. Make further replacement $z_{1} \rightarrow z_{1}+\left(1-z_{1}\right) y$ and $z_{i, p} \rightarrow z_{i, p}+\left(1-z_{i, p}\right) y$ for $i=3, \ldots, n$ and $p=1,2$, then integrate out $z_{1}$ and $y$ using the recursion relations of the beta functions. Finally after performing change of variables $z_{i, p} \rightarrow z_{3,1} z_{i, p}+z_{3,2}\left(1-z_{i, p}\right)$ for $4 \leq i \leq n$ and $p=1,2$ we arrive at (4.19) with $l=3$. 
(ii) The inductive step $l \rightarrow l+1$.

Suppose (4.19) is true for an $l$ such that $3 \leq l<n-2$ and set $z_{l, 1}=x$ and $z_{l, 2}=y$. Let $\langle\langle f\rangle\rangle_{l}=\langle f\rangle_{l} /\langle 1\rangle_{l}$ where

$$
\langle f\rangle_{l}=\oint d x d y f(x, y)[x(1-x) y(1-y)]^{-1-\frac{1}{g}}(x-y)^{-1+\frac{2}{g} l} .
$$

We know from the results in appendix that

$$
\begin{aligned}
& \langle\langle x+y\rangle\rangle_{l}=1, \quad\langle\langle x y\rangle\rangle_{l}=\frac{1}{g-2(l-2)}, \\
& \left\langle\left\langle(x-y)^{2}\right\rangle\right\rangle_{l}=\frac{l}{l-1} \frac{g-2(l-1)}{g-2(l-2)} .
\end{aligned}
$$

To prove (4.19) for $l+1$ it suffices to show that

$$
\begin{aligned}
& \left\langle\left\langle f_{l}(x u+y(1-u), x v+y(1-v))\right\rangle_{l}=f_{l+1}(u, v),\right. \\
& \left\langle\left\langle f_{l}\left(\frac{x+y}{2}, \frac{x+y}{2}\right)\right\rangle\right\rangle_{l}=\frac{g l}{4} \\
& \left\langle\left\langle f_{l}\left(\frac{x+y}{2}, x v+y(1-v)\right)\right\rangle\right\rangle_{l}=\frac{g l}{4} \\
& \left\langle\left\langle f_{l}(x, x)+f_{l}(y, y)\right\rangle\right\rangle_{l}=l(g+1-l) \\
& d_{l+1}=d_{l}\left\langle\left\langle(x-y)^{2}\right\rangle\right\rangle_{l}
\end{aligned}
$$

because then we can reach the desired result first by integrating over $x$ and $y$ while taking into account the data in Table $\mathbf{3}$ and then by performing change of variables $z_{i, p} \rightarrow z_{l+1,1} z_{i, p}+z_{l+1,2}\left(1-z_{i, p}\right)$ for $l+2 \leq i \leq n$ and $p=1,2$. The proof of (4.26)(4.28) goes as follows. One finds from (4.25) that eqs.(4.26) and (4.28) are equivalent to the recursion relations

$$
\begin{aligned}
a_{l+1} & =\frac{l g-2(l-1)^{2}}{(l-1)(g-2(l-2))}\left(a_{l}-b_{l}\right) \\
b_{l+1} & =\frac{1}{g-2(l-2)}\left(a_{l}-b_{l}\right) \\
c_{l+1} & =b_{l}+c_{l} \\
d_{l+1} & =\frac{l}{l-1} \frac{g-2(l-1)}{g-2(l-2)} d_{l}
\end{aligned}
$$


whose solutions with the initial conditions $a_{3}=g-1, b_{3}=1, c_{3}=0$ and $d_{3}=2 g-4$ can easily be shown to be given by (4.22) and (4.23). By substituting (4.22) and (4.23) into (4.21) and using (4.25), we obtain (4.27a-c).

Now that (4.19) is true for $l=n-2$ the case $l=n-1$ and then the case $l=n$ can be proven by similar calculations as above.

If we take $l=n$ in (4.19), the remaining integrations over $z_{n, 1}$ and $z_{n, 2}$ can be readily done via the formulas appearing in the above proof to find that

$$
\hat{T}_{s}(z) \propto \frac{g}{2} \sum_{i, j=1}^{n} M_{i j} A_{i j}(z)+\sum_{i=1}^{n} m_{i} B_{i}(z)+d_{n+1} C(z),
$$

which immediately yields (4.3).

- $\mathrm{g}=\mathcal{D}_{n}, k=1$

In this case we need not introduce parafermions and we have

$$
\begin{aligned}
\hat{T}_{s}(z) \propto & \oint d z_{1} \prod_{i=2}^{n-2} d z_{i, 1} d z_{i, 2} d z_{n-1} d z_{n} \\
\times & {\left[\prod_{p=1}^{2}\left(z_{1}-z_{2, p}\right)^{-1}\left(z_{2, p}-z\right)^{-1} \prod_{i=2}^{n-3} \prod_{p, q=1}^{2}\left(z_{i, p}-z_{i+1, q}\right)^{-1}\right.} \\
& \left.\times \prod_{p=1}^{2}\left(z_{n-2, p}-z_{n-1}\right)^{-1}\left(z_{n-2, p}-z_{n}\right)^{-1} \prod_{i=2}^{n-2}\left(z_{i, 1}-z_{i, 2}\right)^{2}\right]^{1+\frac{1}{g}} \\
& \times: \exp \left[i \alpha _ { + } \left\{\sum_{\mu=1, n-1, n} \alpha_{\mu} \cdot\left(\varphi\left(z_{\mu}\right)-\varphi(z)\right)\right.\right. \\
& \left.\left.+\sum_{i=2}^{n-2} \sum_{p=1}^{2} \alpha_{i} \cdot\left(\varphi\left(z_{i, p}\right)-\varphi(z)\right)\right\}\right]
\end{aligned}
$$

Let $A_{i j}(z)$ and $B_{i}(z)$ be as in (4.17) and set $\zeta_{i}=\frac{1}{2}\left(z_{i, 1}+z_{i, 2}\right)$ for $i=3, \ldots, n-2$. Let $f_{l}(u, v)$ 's be defined as in Lemma (4.18). Quite a similar reasoning as in the previous case entails 
Lemma (4.32). For each $l=3,4, \ldots, n-3$,

$$
\begin{aligned}
\hat{T}_{s}(z) & \propto \oint \prod_{i=l}^{n-2} d z_{i, 1} d z_{i, 2} d z_{n-1} d z_{n} \prod_{p=1}^{2}\left(z_{l, p}\left(1-z_{l, p}\right)\right)^{-1-\frac{1}{g}}\left(z_{l, 1}-z_{l, 2}\right)^{-1+\frac{2}{g} l} \\
& \times P_{l}^{\mathcal{D}}\left(z_{\bullet, \bullet}\right) \prod_{\mu=n-1, n} \prod_{p=1}^{2}\left(z_{n-2, p}-z_{\mu}\right)^{-1-\frac{1}{g}} \\
& \times\left[\left(\frac{g}{2} \sum_{\substack{1 \leq i, j \leq n \\
\min (i, j) \leq l-1}} M_{i j} A_{i j}(z)+\sum_{i=1}^{l-1} m_{i} B_{i}(z)\right)+2 \sum_{l \leq i, j \leq n-2} f_{l}\left(\zeta_{i}, \zeta_{j}\right) A_{i j}(z)\right. \\
& +2 \sum_{\mu=n-1, n} \sum_{i=l}^{n-2} f_{l}\left(z_{\mu}, \zeta_{i}\right) A_{i \mu}(z)+\frac{1}{2} \sum_{\mu=n-1, n} f_{l}\left(z_{\mu}, z_{\mu}\right)\left(A_{\mu \mu}+2 B_{\mu}\right)(z) \\
& \left.+f_{l}\left(z_{n-1}, z_{n}\right) A_{n-1, n}(z)+\sum_{i=l}^{n-2} \sum_{p=1}^{2} f_{l}\left(z_{i, p}, z_{i, p}\right) B_{i}(z)\right]
\end{aligned}
$$

where

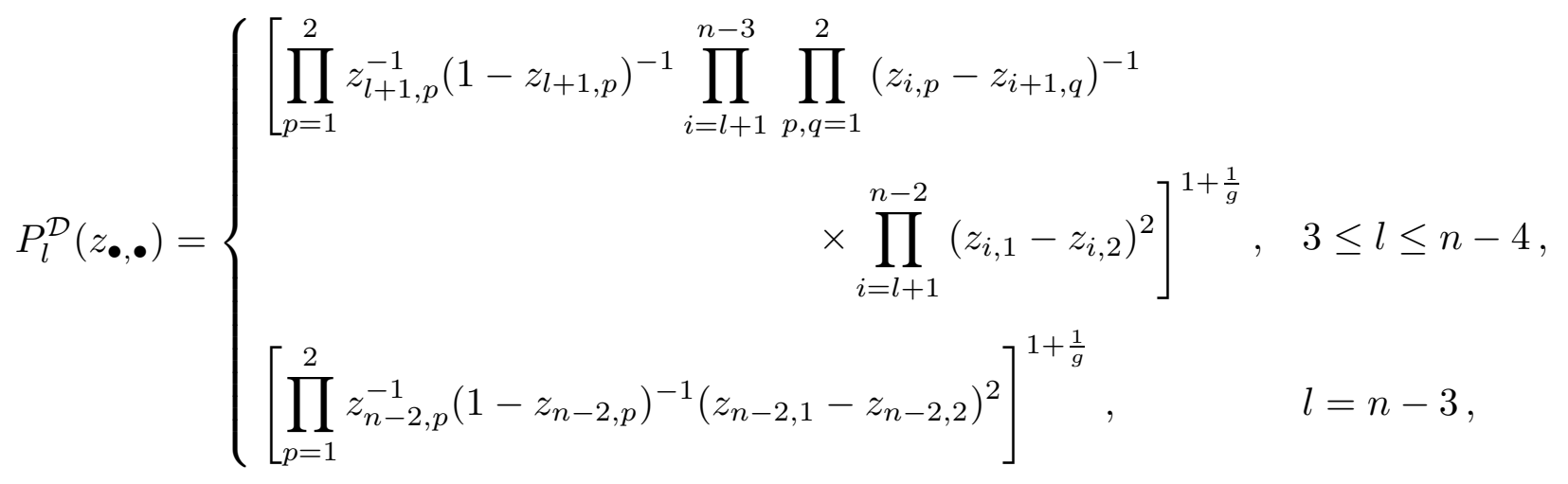

Now set $l=n-3$ in this Lemma, integrate out $z_{n-3,1}$ and $z_{n-3,2}$ and make change of variables $z_{\mu} \rightarrow z_{n-2,1} z_{\mu}+z_{n-2,2}\left(1-z_{\mu}\right)$ for $\mu=n-1, n$. Then further integrating over $z_{n-2,1}$ and $z_{n-2,2}$, we find

$$
\begin{aligned}
\hat{T}_{s}(z) & \propto \oint d z_{n-1} d z_{n}\left[z_{n-1}\left(1-z_{n-1}\right) z_{n}\left(1-z_{n}\right)\right]^{-1-\frac{1}{g}} \\
& \times\left[\left(\frac{g}{2} \sum_{\substack{1 \leq i, j \leq n-2 \\
\min (i, j) \leq n-2}} M_{i j} A_{i j}(z)+\sum_{i=1}^{n-2} m_{i} B_{i}(z)\right)\right.
\end{aligned}
$$




$$
\left.+\frac{1}{2} \sum_{\mu=n-1, n} f_{n-1}\left(z_{\mu}, z_{\mu}\right)\left(A_{\mu \mu}+2 B_{\mu}\right)(z)+f_{n-1}\left(z_{n-1}, z_{n}\right) A_{n-1, n}(z)\right] .
$$

After performing the remaining integrations over $z_{n-1}$ and $z_{n}$, which can be done without difficulty, one ends up with

$$
\hat{T}_{s}(z) \propto \frac{g}{2} \sum_{i, j=1}^{n} M_{i j} A_{i j}(z)+\sum_{i=1}^{n} m_{i} B_{i}(z),
$$

which is equivalent to (4.3).

- $\mathcal{G}_{2}, k=1$

The parafermions in this case can be represented as

$$
\psi_{\alpha}(z)= \begin{cases}\frac{1}{\sqrt{3}} \sum_{j=1}^{3}: \exp \left[i \sqrt{2} \lambda_{j} \cdot X(z)\right]:, & \text { if } \alpha \text { is short } \\ 1, & \text { if } \alpha \text { is long }\end{cases}
$$

where $\lambda_{1}, \lambda_{2}$ and $\lambda_{3}$ are the weights in the vector representation of $s u(3)$ and $X(z)=$ $\left(X_{1}(z), X_{2}(z)\right)$ are free bosons. It is easy to see that if $\alpha$ is a short root

$$
T_{\alpha}^{(\mathrm{pf})}(z)=\frac{1}{3}\left[-: \partial X(z) \cdot \partial X(z)^{*}+\sum_{\beta \in \Delta(s u(3))}: \exp [i \sqrt{2} \beta \cdot X(z)]:\right],
$$

and otherwise $T_{\alpha}^{(\mathrm{pf})}(z)=0$, so that

$$
T^{(\mathrm{pf})}(z)=\frac{c}{d}\left[-: \partial X(z) \cdot \partial X(z)^{-}+\sum_{\beta \in \Delta(s u(3))}: \exp [i \sqrt{2} \beta \cdot X(z)]:\right] .
$$

This representation of parafermions immediately gives

$$
\begin{aligned}
& \hat{T}_{s}(z) \propto \oint \prod_{p=1}^{3} d z_{1, p} \prod_{q=1}^{2} d z_{2, q} \\
& \times\left[\prod_{p=1}^{3} \prod_{q=1}^{2}\left(z_{1, p}-z_{2, q}\right)^{-1} \prod_{q=1}^{2}\left(z_{2, q}-z\right)^{-1}\left(z_{2,1}-z_{2,2}\right)^{2}\right]^{1+\frac{1}{g}} \\
& \times \sum_{j_{1}, j_{2}, j_{3}=1}^{3} \prod_{1 \leq p<q \leq 3}\left(z_{1, p}-z_{1, q}\right)^{2\left(\delta_{j_{p}, j_{q}}+\frac{1}{3 g}\right)}: \exp \left[i \alpha _ { + } \left\{\sum_{p=1}^{3} \alpha_{1} \cdot\left(\varphi\left(z_{1, p}\right)-\varphi(z)\right)\right.\right. \\
& \left.\left.+\sum_{q=1}^{2} \alpha_{2} \cdot\left(\varphi\left(z_{2, q}\right)-\varphi(z)\right)\right\}\right] \because \exp \left[i \sqrt{2} \sum_{p=1}^{3} \lambda_{j_{p}} \cdot \varphi\left(z_{1, p}\right)\right] \because .
\end{aligned}
$$


We first make change of variables $z_{2,1} \rightarrow z+x, z_{2,2} \rightarrow z+x y, z_{1, p} \rightarrow z+x z_{1, p}$ $(p=1,2,3)$, and then integrate over $x$. Further replacement $z_{1, p} \rightarrow z_{1, p}+\left(1-z_{1, p}\right) y$ for $p=1,2,3$ yields

$$
\begin{aligned}
\hat{T}_{s}(z) & \propto \oint d y \prod_{p=1}^{3} d z_{1, p} y^{-\frac{5}{4}}(1-y)^{-\frac{3}{2}} \prod_{p=1}^{3} z_{1, p}^{-\frac{5}{4}}\left(1-z_{1, p}\right)^{-\frac{5}{4}} \prod_{1 \leq p<q \leq 3}\left(z_{1, p}-z_{1, q}\right)^{\frac{1}{6}} \\
& \times\left[\frac{1}{2}\left(\sum_{p=1}^{3}\left\{z_{1, p}+\left(1-z_{1, p}\right) y\right\}\right)^{2} A_{11}(z)\right. \\
& +(1+y) \sum_{p=1}^{3}\left\{z_{1, p}+\left(1-z_{1, p}\right) y\right\} A_{12}(z)+\frac{1}{2}(1+y)^{2} A_{22}(z) \\
& +\sum_{p=1}^{3}\left\{z_{1, p}+\left(1-z_{1, p}\right) y\right\}^{2} B_{1}(z)+\left(1+y^{2}\right) B_{2}(z) \\
& \left.+\frac{1}{6}(1-y)^{2} \sum_{1 \leq p<q \leq 3}\left(z_{1, p}-z_{1, q}\right)^{2} C(z)\right]
\end{aligned}
$$

where

$$
\begin{aligned}
& A_{11}(z)=:\left(i \alpha_{+} \alpha_{1} \cdot \partial \varphi(z)\right)^{2 *} \cdot \frac{1}{3}:(i \partial X(z))^{2}:, \\
& A_{12}(z)=:\left(i \alpha_{+} \alpha_{1} \cdot \partial \varphi(z)\right) \cdot\left(i \alpha_{+} \alpha_{2} \cdot \partial \varphi(z)\right)_{:}, \\
& A_{22}(z)=:\left(i \alpha_{+} \alpha_{2} \cdot \partial \varphi(z)\right)^{2 \cdot}, \\
& B_{1}(z)=\frac{1}{2} i \alpha_{+} \alpha_{1} \cdot \partial^{2} \varphi(z)+\frac{1}{2}:(i \partial X(z))^{2 \cdot}, \quad B_{2}(z)=\frac{1}{2} i \alpha_{+} \alpha_{2} \cdot \partial^{2} \varphi(z), \\
& C(z)=\sum_{\beta \in \Delta(s u(3))}: \exp [i \sqrt{2} \beta \cdot X(z)]_{:}:
\end{aligned}
$$

The integrations over $y$ and $z_{1, p}(p=1,2,3)$ can be done by use of the formulae in appendix leading to

$$
\hat{T}_{s}(z) \propto \frac{g}{2} \sum_{i, j=1}^{2} M_{i j} A_{i j}(z)+\sum_{i=1}^{2} m_{i} B_{i}(z)+C(z) .
$$

This can readily be shown to be equivalent to (4.3) with $T^{(\mathrm{pf})}(z)$ given by (4.39). 


\subsection{Topological Conformal Field Theories - Physical Observables and Correlation} Functions

We now turn to the problem of finding physical observables and their correlation functions. A prerequisite for some field to be a physical observable would be the nullity of its conformal weight with respect to $\hat{T}(z)$. We can easily spell out such fields among $\Psi_{\nu}^{\Lambda, \mu}(z)$ 's:

$$
\bigcup_{w \in W} \mathcal{N}_{w}, \quad \text { where } \quad \mathcal{N}_{w}=\left\{\Psi_{w(\Lambda+\rho)-\rho}^{\Lambda, w(\Lambda+\rho)-\rho}(z), \quad \Lambda \in P_{+}^{k}\right\} .
$$

Notice that these set of fields can be obtained from the Ramond ground fields in sect.3 by simply shifting the exponential factors of the bosons. As a consequence of this, the charge conjugation symmetry of the Ramond ground states translates into the conjugation symmetry under the background charge $-2 \alpha_{0} \rho$, i.e. $q \Leftrightarrow 2 \alpha_{0} \rho-q$. More explicitly,

$$
\Psi_{w(\Lambda+\rho)-\rho}^{\Lambda, w(\Lambda+\rho)-\rho}(z) \underset{\text { conjugation }}{\Longleftrightarrow} \Psi_{w w_{0}(\bar{\Lambda}+\rho)-\rho}^{\bar{\Lambda}, w w_{0}(\bar{\Lambda}+\rho)-\rho}(z)
$$

and hence the conjugate set of $\mathcal{N}_{w}$ is $\mathcal{N}_{w w_{0}}$. If we could adopt the set $\mathcal{N}_{w} \cup \mathcal{N}_{w w_{0}}$ for a fixed $w \in W$ as physical observables of our topological field theory, we would meet no difficulty in finding correlation functions since due to the existence of the charge conjugation under the background charge $-2 \alpha_{0} \rho$, we can for instance consider

$$
\left\langle\Psi_{w(\Lambda+\rho)-\rho}^{\Lambda, w(\Lambda+\rho)-\rho} \Psi_{w w_{0}(\bar{\Lambda}+\rho)-\rho}^{\bar{\Lambda}, w w_{0}(\bar{\Lambda}+\rho)-\rho}\right\rangle_{-2 \alpha_{0} \rho}=1,
$$

However there exists a nuisance in this. In general, two fields in $\mathcal{N}_{w} \cup \mathcal{N}_{w w_{0}}$ are not relatively local and hence it will not be possible to equip $\mathcal{N}_{w} \cup \mathcal{N}_{w w_{0}}$ with a good ring structure.

We will consider in the following

$$
\mathcal{N}_{w=\mathrm{id}}=\left\{\Phi_{\Lambda}(z)=\Psi_{\Lambda}^{\Lambda, 0}(z) \mid \Lambda \in P_{+}^{k}\right\}=\mathcal{R}_{w=\mathrm{id}},
$$

as physical observables. A nice thing about $\mathcal{N}_{w=\text { id }}$ is that its elements $\Phi_{\Lambda}, \Lambda \in P_{+}^{k}$ form a ring and obey

$$
\oint_{0} d z G^{\alpha_{i}}(z) \Phi_{\Lambda}(0)=0, \quad i=1, \ldots, n,
$$

as we have seen in sect.3. On the other hand this choice of physical observables apparently breaks conjugation symmetry and hence we have to devise a procedure to make viable correlation functions. If we have a legitimate procedure to make correlation functions of 
$\Phi_{\Lambda}$ 's the conditions (4.48) and (4.3) imply, as in usual topological field theories, that the correlation functions are independent of the world-sheet positions of $\Phi_{\Lambda}$ 's.

Now we wish to tackle the problem of constructing correlation functions among $\Phi_{\Lambda}$ 's to make our topological field theory sensible. But before doing that, we should spend a while to think over what the BRST structure of the theory must be. The problem of finding out the BRST-structure in our theory is essentially the samet as that of extending the results of Felder [20] and others [21] to the coset model $G_{k} \times G_{0} / G_{k}$ using the representation in terms of free bosons and parafermions. The latter problem is a formidable task and as far as we know there seems to have been no complete analysis yet. Here we can only make several conjectural statements which we admit are not based on a rigorous footing. Let $\mathcal{F}_{\Lambda, \Lambda^{\prime}}^{\beta}$ be the space of the fields with parafermionic charge $\beta$ and $u(1)^{n}$ charge $-\frac{1}{\alpha_{+}}\left(\alpha_{+} \Lambda+\alpha_{-} \Lambda^{\prime}\right)$. The 'BRST charges' or the intertwiners

$$
Q_{12}: \mathcal{F}_{\Lambda_{1}, \Lambda_{1}^{\prime}}^{\beta_{1}} \longrightarrow \mathcal{F}_{\Lambda_{2}, \Lambda_{2}^{\prime}}^{\beta_{2}}
$$

will be integrals of products of $G^{\alpha_{i}}(z)$ 's but their explicit form must be dependent on $\mathcal{F}_{\Lambda_{1}, \Lambda_{1}^{\prime}}^{\beta_{1}}$ and $\mathcal{F}_{\Lambda_{2}, \Lambda_{2}^{\prime}}^{\beta_{2}}$. The spaces $\mathcal{F}_{\Lambda, \Lambda^{\prime}}^{\beta}$ and their intertwiners determine a directed graph which we suppose coincides with the Hasse diagram of the affine Weyl group of $\mathbf{g}$. We conjecture that a part of this diagram is given by

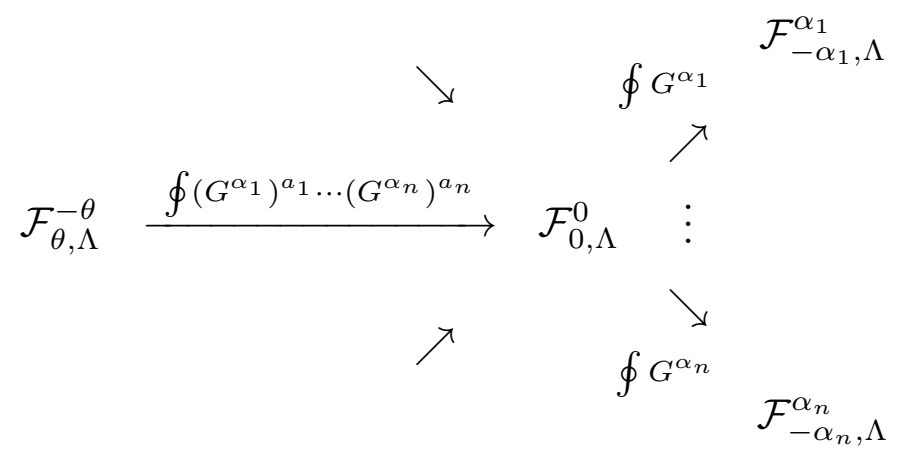

where we are again sloppy about the contours and the normalization constants of the intertwiners. For instance we have $\hat{T}(z) \in \mathcal{F}_{0,0}^{0}$ and $G^{-\theta}(z) \in \mathcal{F}_{\theta, 0}^{-\theta}$. If we could complete the diagram with the explicit knowledge of the intertwiners, we could construct the BRST complex and discuss its cohomology. As mentioned above this is far beyond the scope of the present paper. Our physical observables $\Phi_{\Lambda}$ 's are in $\mathcal{F}_{0, \Lambda}^{0}$ and annihilated by $\oint G^{\alpha_{i}}$ 's. We expect them to be non-trivial cocycles of the BRST complex.

4 However there is a slight difference between the two models as we shall explain shortly. 
The difference between our topological field theory and the coset model $G_{k} \times G_{0} / G_{k}$ resides in the following points. In the conventional $G_{k} \times G_{0} / G_{k}$ model the generators of the chiral algebra are Weyl-invariant (like the stress-energy tensor or $\mathcal{W}$ generators) and in particular $u(1)^{n}$-charges are not considered as quantum numbers. Consequently all the $\mathcal{N}_{w}$ 's with different $w$ are physically identified and the 'Kac table' is labeled solely by $P_{+}^{k}$. It is believed, however, that in practical calculations of correlation functions one may take any convenient representatives from appropriate $\mathcal{N}_{w}$ 's so as to minimize introductions of the screening charges. On the other hand our topological field theory cares about $u(1)^{n}$ quantum numbers since it was obtained by twisting the higher-rank supersymmetric models which have $u(1)^{n}$ symmetry. Therefore we have to consider correlation functions strictly within $\mathcal{N}_{\text {id }}$ once we choose $\mathcal{N}_{\text {id }}$ as physical observables.

Since, by this restriction of physical observables, the original conjugation symmetry is lost and it is inevitable to introduce

$$
\oint d z S^{\alpha_{i}}(z), \quad \text { where } S^{\alpha_{i}}(z)=\psi_{-\alpha_{i}}(z)^{*} \exp \left[i \alpha_{-} \alpha_{i} \cdot \varphi(z)\right]^{*},
$$

which however do not break the BRST invariance since

$$
\left[\oint d z G^{\alpha_{i}}(z), \oint d z S^{\alpha_{j}}(z)\right]=0
$$

by virtue of

$$
G^{\alpha_{i}}(z) S^{\alpha_{i}}\left(z^{\prime}\right)=\frac{1}{\left(z-z^{\prime}\right)^{2}} \cdot e^{i \alpha_{0} \alpha_{i} \cdot \varphi\left(z^{\prime}\right)} \cdot+\frac{\alpha_{+}}{\alpha_{0}} \frac{1}{z-z^{\prime}} \partial_{z^{\prime}}\left(\cdot e^{i \alpha_{0} \alpha_{i} \cdot \varphi\left(z^{\prime}\right) \cdot} \cdot\right)+\cdots
$$

and can be used to balance the excess of the $u(1)^{n}$ charge caused by products of $\Phi_{\Lambda}$ 's. For instance,

$$
\left\langle\left(\oint S^{\alpha_{1}}\right)^{m_{1}} \cdots\left(\oint S^{\alpha_{n}}\right)^{m_{n}} \Phi_{\Lambda} \Phi_{\bar{\Lambda}}\right\rangle=1
$$

where

$$
\Lambda+\bar{\Lambda}=\Lambda-w_{0}(\Lambda)=\sum_{i=1}^{n} m_{i} \alpha_{i}
$$

In this way by inserting $\oint S^{\alpha_{i}}$ 's one can consider an arbitrary correlation functions of $\Phi_{\Lambda}$ 's and hence one is able to formulate a consistent topological field theory (modulo some technical difficulties). 
According to the general formalism of topological field theory [4, 5,22], if we have BRST-invariant operators of $(0,0)$-form there exist $(1,1)$-forms (or $(1,0)$-forms if we restrict ourselves to the left chiral part) related via 'descent equations' whose integrals become again BRST-invariant. Introduction of these extra physical observables can formally be interpreted as perturbations of the original topological field theory by the (1,1)-form operators. An analogous situation arises here for our topological field theory obtained above. Let

$$
\Psi_{\Lambda}(z)=\phi_{\Lambda-\theta}^{\Lambda}(z)^{*} \exp \left[-i\left(\alpha_{+} \theta+\alpha_{-} \Lambda\right) \cdot \varphi(z)\right]^{*}
$$

for $\Lambda \in P_{+}^{k}$. It is easy to see that $\Psi_{\Lambda}(z)$ has a conformal weight 1 with respect to $\hat{T}(z)$ and $\Psi_{\Lambda}(z) \in \mathcal{F}_{\theta, \Lambda}^{-\theta}$. Furthermore we have

$$
G^{-\theta}(z) \Phi_{\Lambda}(w)=\frac{1}{z-w} \Psi_{\Lambda}(w)+\cdots
$$

and hence

$$
\begin{aligned}
& \oint\left(G^{\alpha_{1}}\right)^{a_{1}} \cdots\left(G^{\alpha_{n}}\right)^{a_{n}} \oint_{0} d w \Psi_{\Lambda}(w) \\
& =\oint\left(G^{\alpha_{1}}\right)^{a_{1}} \cdots\left(G^{\alpha_{n}}\right)^{a_{n}} \oint_{0} d w \oint_{w} d z G^{-\theta}(z) \Phi_{\Lambda}(w) \\
& \propto \oint_{0} d w \oint_{w} d z \hat{T}(z) \Phi_{\Lambda}(w)=0,
\end{aligned}
$$

which shows that $\oint_{0} d w \Psi_{\Lambda}(w), \Lambda \in P_{+}^{k}$ are BRST invariant observables provided that the diagram (4.50) is correct.

Here we should comment again on the exceptional case $\mathbf{g}=s u(2)$. We noted in sect.3 that $\mathcal{V}_{w=+1}$ is charge-conjugation symmetric per se, i.e. $\mathcal{V}_{w=+1}=\mathcal{V}_{w=-1}$. Similarly, we have here $\mathcal{N}_{w=+1}=\mathcal{N}_{w=-1}$. In other words the chiral ring $\mathcal{N}_{w=+1}=\mathcal{R}_{w=+1}$ is symmetric under conjugation with background charge $-2 \alpha_{0} \rho$ which is just a manifestation of the Poincaré duality in $N=2$ superconformal field theory. Therefore it is possible to consider correlation functions among the elements of $\mathcal{N}_{w=+1}$ as in (4.46) without introducing an extra machinery. This is the route usually taken in constructing topological field theory from the $N=2$ minimal model [5]. So we can obtain two possible topological field theories starting from the $N=2$ minimal model, the usual one and the one constructed here. They are different theories and this is particularly evident from the fact that their selection rules of physical observables are different.

As a final comment of this section we should like to speculate on a possible connection of our topological field theory to $G / G$ topological field theory. In the existing literature 
[8], $G / G$ theory is formulated by gauging the WZW model. Its physical observables are labeled by $P_{+}^{k}$ as in our theory and it has a marked property to the effect that threepoint functions essentially coincide with the structure constants of the fusion algebra of the WZW model. We suspect that three-point functions in our topological field theory

$$
N_{\Lambda \Lambda^{\prime} \Lambda^{\prime \prime}}=\left\langle\left(\oint S^{\alpha_{1}}\right)^{m_{1}} \cdots\left(\oint S^{\alpha_{n}}\right)^{m_{n}} \Phi_{\Lambda} \Phi_{\Lambda^{\prime}} \Phi_{\Lambda^{\prime \prime}}\right\rangle,
$$

where

$$
\Lambda+\Lambda^{\prime}+\Lambda^{\prime \prime}=\sum_{i=1}^{n} m_{i} \alpha_{i}
$$

may similarly coincide with the fusion rules coefficients of the WZW model and hence the two theories may be equivalent. We remark that a similar idea is proposed when the relation between the twisted $N=2$ Kazama-Suzuki model and the $G / G$ theory is discussed [23]. Unfortunately the explicit evaluation of (4.59) seems quite a formidable task. In addition the two theories are formulated quite differently, one in terms of the gauged WZW model and the other in terms of a combined system of parafermions and free bosons. Thus whether this equivalence is indeed true or not remains open.

\section{Concluding Remarks}

In this paper we have elaborated on the structure of higher-rank supersymmetric models which are natural generalization of the $N=2$ minimal model and have endeavored to construct a class of topological field theories. Although there remain technical problems to be overcome for our topological field theories to become full-fledged, we hope we have convinced the reader that such topological field theories can really exist.

We would like to end this paper with the following remark. When the rank of $\mathrm{g}$ exceeds

one, the existence of the extended Virasoro algebra is expected in our topological field theory. Suppose that the extended algebra is of the $\mathcal{W}$ algebra type, then the $\mathcal{W}$ currents in topological algebra will take the BRST-exact form. Let us report a tiny calculation which exemplifies this idea. We start with a BRST-exact expression

$$
\mathcal{W}(z)=\oint\left(G^{\alpha_{1}}\right)^{a_{1}} \cdots\left(G^{\alpha_{n}}\right)^{a_{n}}: v \cdot J(z) G^{-\theta}(z)^{:} .
$$

It is easy to see that $\mathcal{W}(z)$ is a spin-3 field with respect to $\hat{T}(z)$ provided that

$$
\left(2 \alpha_{0} \rho+\alpha_{+} \theta\right) \cdot v=0 .
$$


In the case $\mathbf{g}=s u(3)$, the condition (5.2) fixes $v$ uniquely up to a multiplicative constant and via an explicit calculation 5 we have confirmed for $k=1$

$$
\begin{gathered}
\mathcal{W}(z) \propto^{*}\left[\left(\partial \varphi_{2}(z)\right)^{3}-3 \partial \varphi_{2}(z)\left(\partial \varphi_{1}(z)\right)^{2}+\frac{3 i}{2 \sqrt{2}}\left(\partial \varphi_{1}(z) \partial^{2} \varphi_{1}(z)-\partial \varphi_{2}(z) \partial^{2} \varphi_{2}(z)\right)\right. \\
\left.+\frac{\sqrt{6} i}{2} \partial \varphi_{2}(z) \partial^{2} \varphi_{1}(z)-\frac{1}{8} \partial^{3} \varphi_{2}(z)+\frac{\sqrt{3}}{8} \partial^{3} \varphi_{1}(z)\right]
\end{gathered}
$$

where the RHS is the standard spin-3 generator of the $\mathcal{W}_{3}$ algebra [24,25]. Thus in this case we obtain the $\mathcal{W}_{3}$ extended topological algebra. It is a frequently asked but never completely answered question precisely in what way the extended chiral algebra such as the $\mathcal{W}$ algebra and the idea of topological conformal field theory can be combined. We hope our higher-rank topological models can shed a new light on this issue in the future.

\section{Acknowledgements}

One of us (SKY) would like to thank H. Saleur for discussions and warm hospitality at Yale University where part of this work was done.

\section{Appendix. Selberg-type Integrals}

In this appendix we gather useful formulas for integrals of the form $\mathrm{B}$

$$
\mathcal{J}^{(N)}\left(a_{1}, \ldots, a_{N} ; b ; c\right)=\oint \prod_{i=1}^{N} d z_{i} \prod_{i=1}^{N} z_{i}^{a_{i}-1}\left(1-z_{i}\right)^{b-1} \prod_{i<j}^{N}\left(z_{i}-z_{j}\right)^{2 c}
$$

where the integration contour must be closed and such that it avoids the singularities of the integrand. It is easy to show that

$$
\sum_{i=1}^{N}\left[\left(a_{i}+m\right)\left\langle z_{i}^{m}\right\rangle-(b-1)\left\langle\frac{z_{i}^{m+1}}{1-z_{i}}\right\rangle\right]+\sum_{i<j}^{N} 2 c\left\langle\frac{z_{i}^{m+1}-z_{j}^{m+1}}{z_{i}-z_{j}}\right\rangle=0,
$$

where we introduced the notation,

$$
\left\langle f\left(z_{1}, \ldots, z_{N}\right)\right\rangle=\oint \prod_{i=1}^{N} d z_{i} \prod_{i=1}^{N} z_{i}^{a_{i}-1}\left(1-z_{i}\right)^{b-1} \prod_{i<j}^{N}\left(z_{i}-z_{j}\right)^{2 c} f\left(z_{1}, \ldots, z_{N}\right),
$$

5 with the aid of Mathematica

6 cf. refs. 26,27. 
for any $f\left(z_{1}, \ldots, z_{N}\right)$. By setting $m=0$ in (A.2) we find

$$
(b-1) \sum_{i=1}^{N}\left\langle\left\langle\frac{1}{1-z_{i}}\right\rangle\right\rangle=\sum_{i=1}^{N} a_{i}+N(b-1)+N(N-1) c
$$

where $\left\langle\left\langle f\left(z_{1}, \ldots, z_{N}\right)\right\rangle\right\rangle:=\left\langle f\left(z_{1}, \ldots, z_{N}\right)\right\rangle /\langle 1\rangle$. Using (A.4), eq.(A.2) can be rewritten as

$$
\begin{aligned}
\sum_{i=1}^{N}\left[\left(a_{i}+m\right)\right. & \left.\left\langle\left\langle z_{i}^{m}\right\rangle\right\rangle+(b-1)\left\langle\left\langle\frac{1-z_{i}^{m+1}}{1-z_{i}}\right\rangle\right\rangle\right] \\
& +\sum_{i<j}^{N} 2 c\left\langle\left\langle\frac{z_{i}^{m+1}-z_{j}^{m+1}}{z_{i}-z_{j}}\right\rangle\right\rangle=\sum_{i=1}^{N} a_{i}+N(b-1)+N(N-1) c .
\end{aligned}
$$

When $m$ is a positive integer greater than 1 , this becomes

$$
\begin{gathered}
\sum_{i=1}^{N}\left[a_{i}+b+2(N-1) c+m-1\right]\left\langle\left\langle z_{i}^{m}\right\rangle\right\rangle+(b-1) \sum_{i=1}^{N} \sum_{p=1}^{m-1}\left\langle\left\langle z_{i}^{p}\right\rangle\right\rangle \\
+2 c \sum_{i<j}^{N} \sum_{p=1}^{m-1}\left\langle\left\langle z_{i}^{p} z_{j}^{m-p}\right\rangle\right\rangle=\sum_{i=1}^{N} a_{i}+N(N-1) c,
\end{gathered}
$$

while in the case $m=1$ we find

$$
\sum_{i=1}^{N}\left(a_{i}+b+2(N-1) c\right)\left\langle\left\langle z_{i}\right\rangle\right\rangle=\sum_{i=1}^{N} a_{i}+N(N-1) c .
$$

For a fixed $j$ such that $1 \leq j \leq N$ replace $a_{j}$ by $a_{j}+1$ in (A.7). Then we obtain

$$
\begin{aligned}
\sum_{i \neq j}^{N}\left(a_{i}+b+2(N-1) c\right)\left\langle\left\langle z_{i} z_{j}\right\rangle\right\rangle & +\left(a_{j}+b+2(N-1) c+1\right)\left\langle\left\langle z_{j}^{2}\right\rangle\right\rangle \\
& =\left[\sum_{i=1}^{N} a_{i}+N(N-1) c+1\right]\left\langle\left\langle z_{j}\right\rangle\right\rangle .
\end{aligned}
$$

Eqs. (A.6) with $m=2$ and (A.8) produce

$$
\begin{aligned}
\sum_{i<j}^{N}\left[a_{i}+a_{j}+\right. & 2 b+2(2 N-3) c]\left\langle\left\langle z_{i} z_{j}\right\rangle\right\rangle \\
= & {\left[\sum_{j=1}^{N} a_{j}+b+N(N-1) c\right] \sum_{i=1}^{N}\left\langle\left\langle z_{i}\right\rangle\right\rangle-\left[\sum_{i=1}^{N} a_{i}+N(N-1) c\right] . }
\end{aligned}
$$


If we take $a_{i}=a$ for all $i(i=1, \ldots, N)$, it then easily follows that

$$
\begin{aligned}
& \sum_{i=1}^{N}\left\langle\left\langle z_{i}\right\rangle\right\rangle=\frac{N(a+(N-1) c)}{a+b+2(N-1) c} \\
& \sum_{i<j}^{N}\left\langle\left\langle z_{i} z_{j}\right\rangle\right\rangle=\frac{\frac{1}{2} N(N-1)(a+(N-1) c)(a+(N-2) c)}{(a+b+(2 N-2) c)(a+b+(2 N-3) c)} \\
& \sum_{i=1}^{N}\left\langle\left\langle z_{i}\left(1-z_{i}\right)\right\rangle\right\rangle \\
& \quad=\frac{N(a+(N-1) c)(b+(N-1) c)(a+b+(N-2) c)}{(a+b+2(N-1) c+1)(a+b+2(N-1) c)(a+b+(2 N-3) c)}
\end{aligned}
$$

and

$$
\begin{aligned}
\sum_{i<j}^{N}\left\langle\left\langle\left(z_{i}-z_{j}\right)^{2}\right\rangle\right\rangle & =(N-1) \sum_{i=1}^{N}\left\langle\left\langle z_{i}^{2}\right\rangle\right\rangle-2 \sum_{i<j}^{N}\left\langle\left\langle z_{i} z_{j}\right\rangle\right\rangle \\
& =\frac{N(N-1)(N c+1)(a+(N-1) c)(b+(N-1) c)}{(a+b+2(N-1) c+1)(a+b+2(N-1) c)(a+b+(2 N-3) c)} .
\end{aligned}
$$

For $N=1, \mathcal{J}^{(N)}\left(a_{1}, \ldots, a_{N} ; b ; c\right)$ reduces to the beta function $B(a, b)$ and eqs.(A.10a) and (A.10d) become the well-known recursion formulas,

$$
\begin{aligned}
& B(a+1, b)=\frac{a}{a+b} B(a, b) \\
& B(a+1, b+1)=\frac{a b}{(a+b)(a+b+1)} B(a, b) .
\end{aligned}
$$




\section{References}

[1] A.B. Zamolodchikov and V.A. Fateev, Sov. Phys. JETP. 63 (1986) 913

[Zh. Eksp. Teor. Fiz. 90 (1986) 1553];

W. Boucher, D. Friedan and A. Kent, Phys. Lett. B172 (1986) 316;

P. Di Vecchia, J.L. Petersen, M. Yu and H.B. Zheng, Phys. Lett. B174 (1986) 280;

Z. Qiu, Phys. Lett. B188 (1987) 207.

[2] T. Kawai, Phys. Lett. B259 (1991) 460; B261 (1991) 520E.

[3] T. Eguchi, T. Kawai, S. Mizoguchi and S.-K. Yang, KEK-TH-303, KEK preprint 91-111, (September 1991), to appear in Rev. Math. Phys.

[4] E. Witten, Commun. Math. Phys. 118 (1988) 411; Nucl. Phys. B340 (1990) 281.

[5] T. Eguchi and S.-K. Yang, Mod. Phys. Lett. A4 (1990) 1653.

[6] W. Lerche, Phys. Lett. B252 (1990) 349.

[7] T. Eguchi, S. Hosono and S.-K. Yang, Commun. Math. Phys. 140 (1991) 159.

[8] K. Gawedzki and A. Kupiainen, Nucl. Phys. B320 (1989) 625;

H. Verlinde, Nucl. Phys. B337 (1990) 652;

E. Witten, Commun. Math. Phys. 144 (1992) 189;

K. Intriligator, Mod. Phys. Lett. A6 (1991) 3543;

M. Spiegelglas and S. Yankielowicz, TECHNION-PH-90-34.

[9] T. Kawai, T. Uchino and S.-K. Yang, KEK-TH-311, KEK preprint 91-190, (December 1991), to appear in Prog. Theor. Phys. Suppl.

[10] A.B. Zamolodchikov and V.A. Fateev, Sov. Phys. JETP. 62 (1985) 215

[Zh. Eksp. Teor. Fiz. 89 (1985) 380].

[11] M. Ninomiya and K. Yamagishi, Phys. Lett. B183 (1987) 323.

[12] D. Gepner, Nucl. Phys. B290 (1987) 10.

[13] G. Dunne, I. Halliday and P. Suranyi, Nucl. Phys. B325 (1989) 526.

[14] P. Goddard, N. Nahm, D. Olive and A. Schwimmer, Commun. Math. Phys. 107 (1986) 179.

[15] N. Bourbaki, Groupes et algèbres de Lie, (Masson, Paris, 1981).

[16] V.G. Kac and D.H. Peterson, Adv. Math. 53 (1984) 125.

[17] A. Schwimmer and N. Seiberg, Phys. Lett. B184 (1987) 191.

[18] W. Lerche, C. Vafa and N.P. Warner, Nucl. Phys. B324 (1989) 427.

[19] A.B. Zamolodchikov and V.A. Fateev, Theor. Math. Phys. 71 (1988) 451 [Teor. Mat. Fiz. 71 (1987) 163].

[20] G. Felder, Nucl. Phys. B317 (1989) 215.

[21] P. Bouwknegt, J. McCarthy and K. Pilch, Commun. Math. Phys. 131 (1990) 125;

S. Mizoguchi and T. Nakatsu, Tokyo preprint, UT566, (September 1990);

S.-w. Chung, E. Lyman and S.-H. H. Tye, Cornell preprint, CLNS 91/1057, (May 1991). 
[22] R. Dijkgraaf, E. Verlinde and H. Verlinde, Nucl. Phys. B352 (1991) 59; PUPT-1217, IASSNS-HEP-90-80, (November, 1990).

[23] D. Nemeschansky and N.P. Warner, USC-91/031, (October, 1991).

[24] V.A. Fateev and A.B. Zamolodchikov, Nucl. Phys. B280 [FS18] (1987) 644.

[25] L.J. Romans, Nucl. Phys. B352 (1991) 829.

[26] Vl.S. Dotsenko and V.A. Fateev, Nucl. Phys. B251 (1985) 691.

[27] K. Aomoto, in Ramanujuan Revisited, Proceedings of the Centenary Conference, Eds. G.F. Andrews et al., (Academic Press, New York, 1988). 


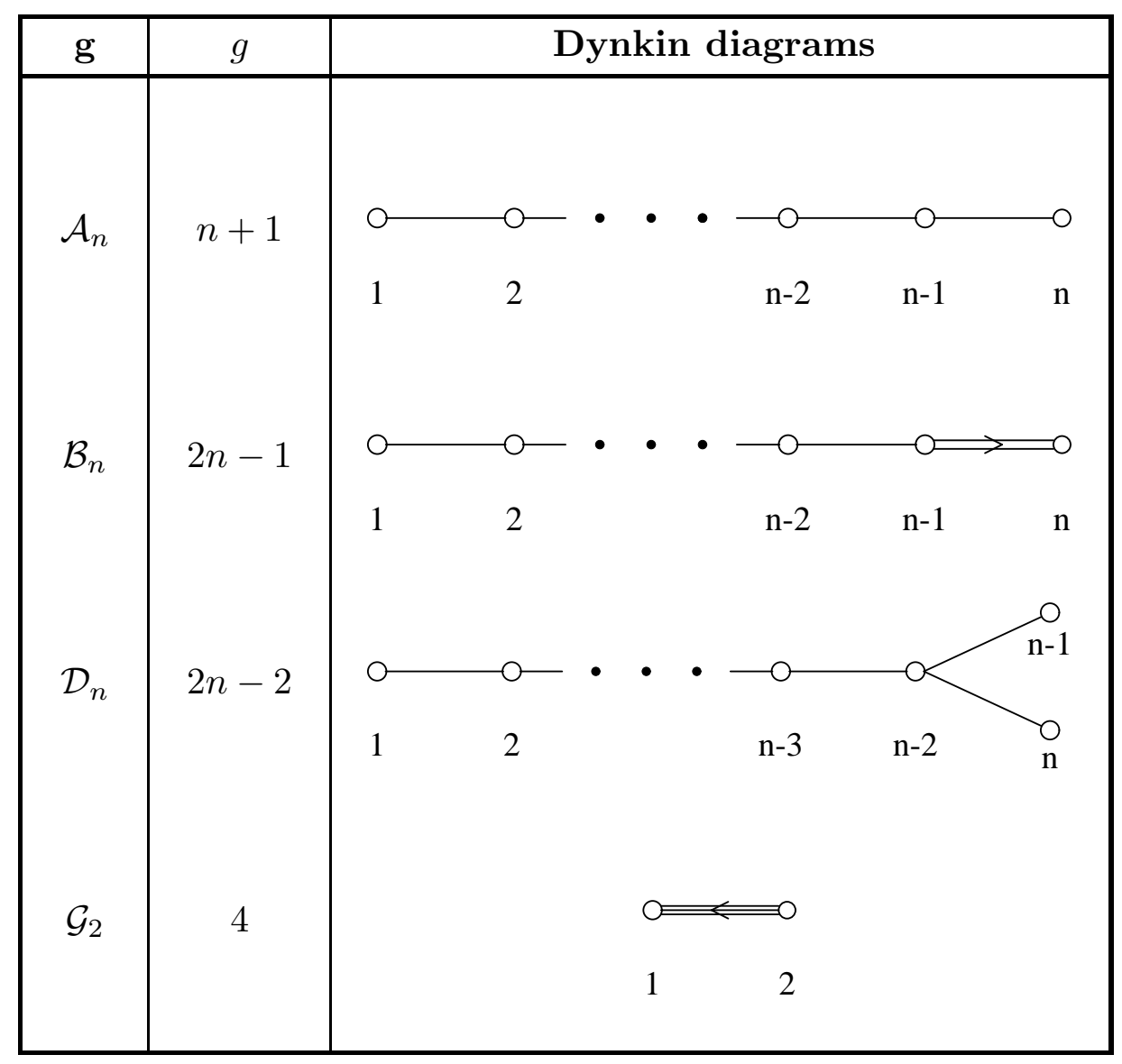

Table 1. Relevant Dynkin diagrams and the numbering of their nodes. 


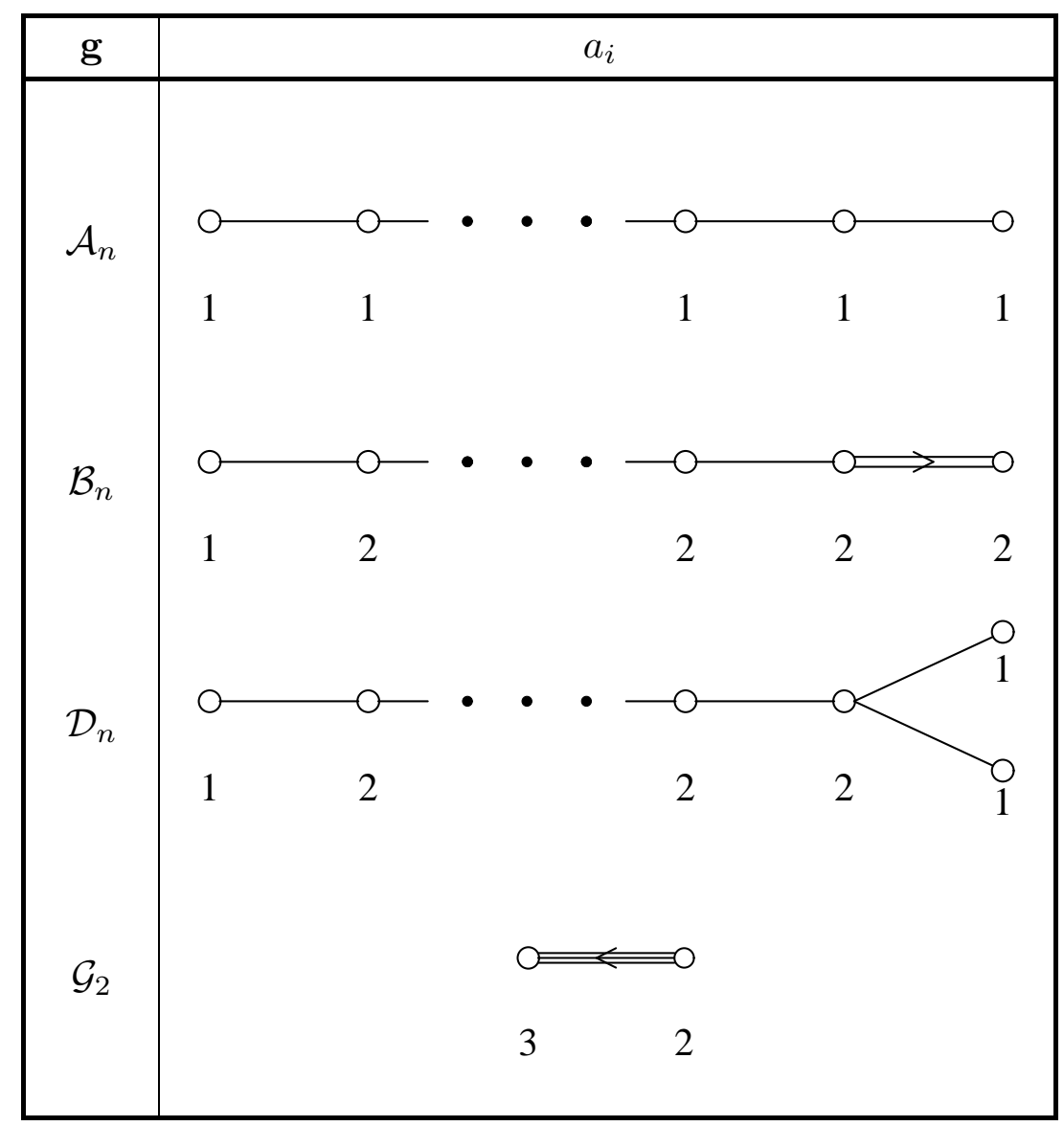

Table 2. List of $a_{i}$ 's where $\theta=\sum_{i=1}^{n} a_{i} \alpha_{i}$. 


\begin{tabular}{|c|c|c|}
\hline $\mathrm{g}$ & $M_{i j} \quad(1 \leq i \leq j \leq n)$ & $2 \rho=\sum_{i=1}^{n} m_{i} \alpha_{i}$ \\
\hline $\mathcal{A}_{n}$ & $M_{i j}=\frac{i(n+1-j)}{n+1}$ & $2 \rho=\sum_{i=1}^{n} i(n+1-i) \alpha_{i}$ \\
\hline $\mathcal{B}_{n}$ & $M_{i j}=i$ & $2 \rho=\sum_{i=1}^{n} i(2 n-i) \alpha_{i}$ \\
\hline $\mathcal{D}_{n}$ & $\begin{array}{l}M_{i j}=i, \quad 1 \leq i, j \leq n-2 \\
M_{i, n-1}=M_{i n}=\frac{i}{2}, \quad 1 \leq i \leq n-2 \\
M_{n-1, n-1}=M_{n n}=\frac{n}{4} \\
M_{n-1, n}=\frac{n-2}{4}\end{array}$ & $\begin{aligned} 2 \rho & =\sum_{i=1}^{n-2} 2\left(i n-\frac{i(i+1)}{2}\right) \alpha_{i} \\
& +\frac{n(n-1)}{2}\left(\alpha_{n-1}+\alpha_{n}\right)\end{aligned}$ \\
\hline $\mathcal{G}_{2}$ & $M_{11}=6, \quad M_{12}=3, \quad M_{22}=2$ & $2 \rho=10 \alpha_{1}+6 \alpha_{2}$ \\
\hline
\end{tabular}

Table 3. Some useful Lie algebraic data. 